\title{
Directed Energy Technology Overview
}

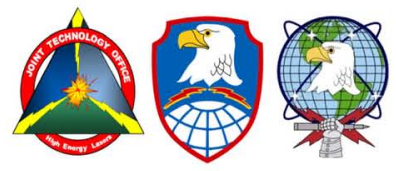

Dr. Michael Lavan, Director Emerging Technology Directorate and Mr. John Wachs, Senior Engineer

Directed Energy Division, Space and

Missile Defense Technical Center

USASMDCIARSTRAT

Distribution A - This document has been approved for public release with unlimited distribution. Reference SMDC/ARSTRAT public release approval case number 1199, 2 June 2011. 
U.S. Almony Space and Missille Defense CommumdV

\section{Outline}

- Laser C-RAM Operational Concept

- Tactical High Energy Laser (THEL) Demonstrated C-RAM

- High Energy Solid State Laser Technology Thrust Areas

- Joint High Power Solid State Laser Program

- Mobile HEL Technology Demonstration Program (HELTD)

- Solid State Laser Technology Integration Experiment (SSLTE)

- Robust Electric Laser Initiative (RELI)

- Light Weight Beam Director (LWBD) Program

- High Power Microwave Efforts

- Summary 


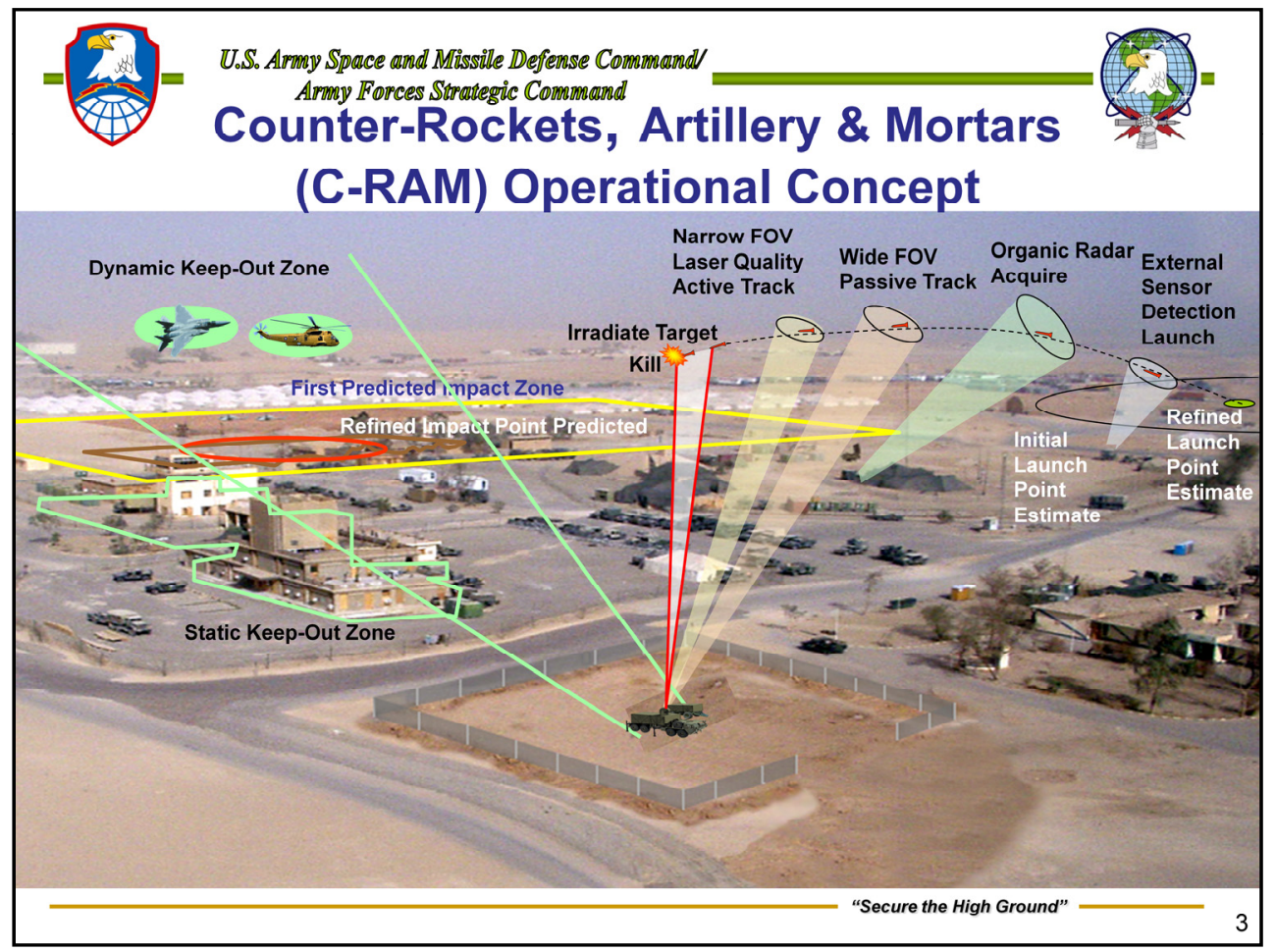

Typically, for a RAM target, a radar will detect and acquire the target soon after line of sight is established with the object. As demonstrated on THEL, the radar track can be handed off to the laser weapon system on board optical sensors.

The Wide field of view, Passive Track Sensor of the beam control system (BCS) receives the handover instructions from the radar, and must then slew to that point in space and attempt to optically acquire the target in its FOV. Typically, this will occur within several seconds.

For the narrow field of view, which is the Laser Quality Active Track Sensor, tracking normally starts soon after the Wide FOV sensor acquires the target. Irradiation of the target can begin immediately after the target is within the Narrow FOV Tracker. However, for longer range targets, it may be prudent to delay putting the beam on the target in order to achieve a more efficient kill.

Also shown are other activities related to the engagement of airborne targets. First, control measures, called keep-out zones are established to protect friendly assets. These are both static, which may set a limit on the minimum elevation angle that a target can be engaged; or dynamic, where friendly airborne targets are tracked through a situational air picture, and the high energy laser is not permitted to operate when the beam would intercept one of these dynamic keep-out zones.

Also shown are other functions that the BMC3 system of the HEL platform would provide. First, based on early track information it would calculate an initial launch point estimate and predicted impact point. The former is used by forces in an attempt to eliminate the launch point and the latter to assist in the decision process of whether to engage that object. Both of these calculations are refined as additional track information becomes available.

C-UAV operations will involve similar operations, but the ranges could be much greater and the targets could be moving more slowly. 


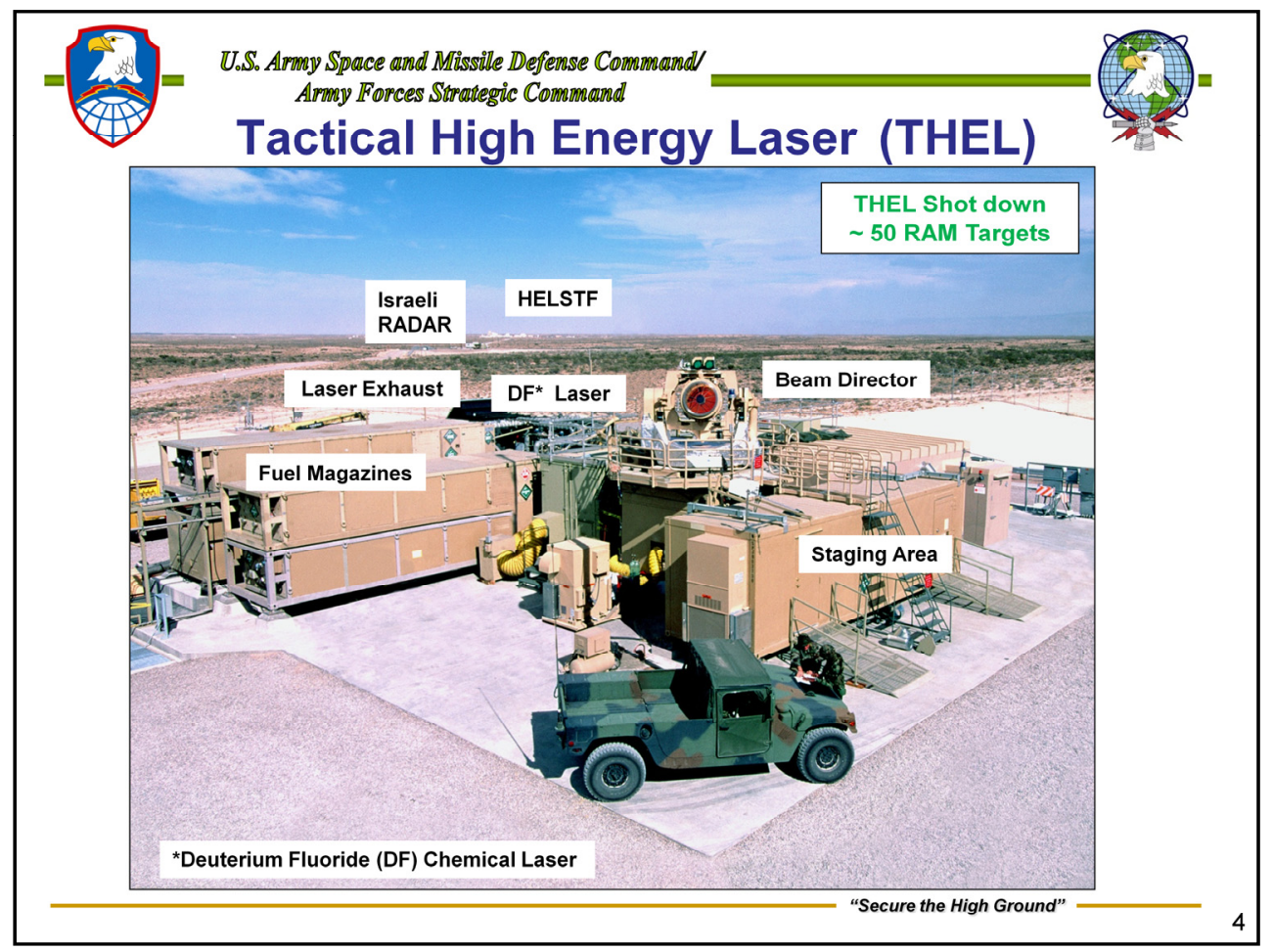

The Tactical High-Energy Laser (THEL) program was a US/Israeli cooperative research and development effort to develop a laser for military use to counter terrorist rocket attacks. The THEL was also referred to as the Nautilus laser system in Israel. The program was also designated an Advanced Concept Technology Demonstration (ACTD) by the Secretary of Defense with Presidential interest.

The THEL utilized mature deuterium fluoride (DF) chemical laser technologies. The system was designed and built under an SMDC/ARSTRAT prime contract awarded to TRW (now Northrop Grumman Corporation) in 1996. For primarily Israeli applications, the original THEL was designed as a fixed location, which eliminated most weight, size and power restrictions.

In 2000 and 2001, THEL successfully shot down 28 Katyusha rockets and 5 artillery shells. During a test conducted on August 24, 2004 the system successfully shot down multiple mortar rounds. The tests represented actual mortar threat scenarios including both single mortar rounds and salvos that were intercepted by the THEL testbed and destroyed.

The fixed site system was not compatible with the fluid, mobile nature of modern combat. A mobile version (MTHEL) the size of three large semi trailers was designed, which could possibly be downsized to a single semi trailer size, depending on mission requirements. However, doing this while maintaining the original THEL performance characteristics would be difficult. Furthermore, the Israeli government, which had been providing significant funding, decreased their financial support in 2004, resulting in the cancellation of the MTHEL program and elimination of additonal investment in DF chemical laser technologies by the US Army. 


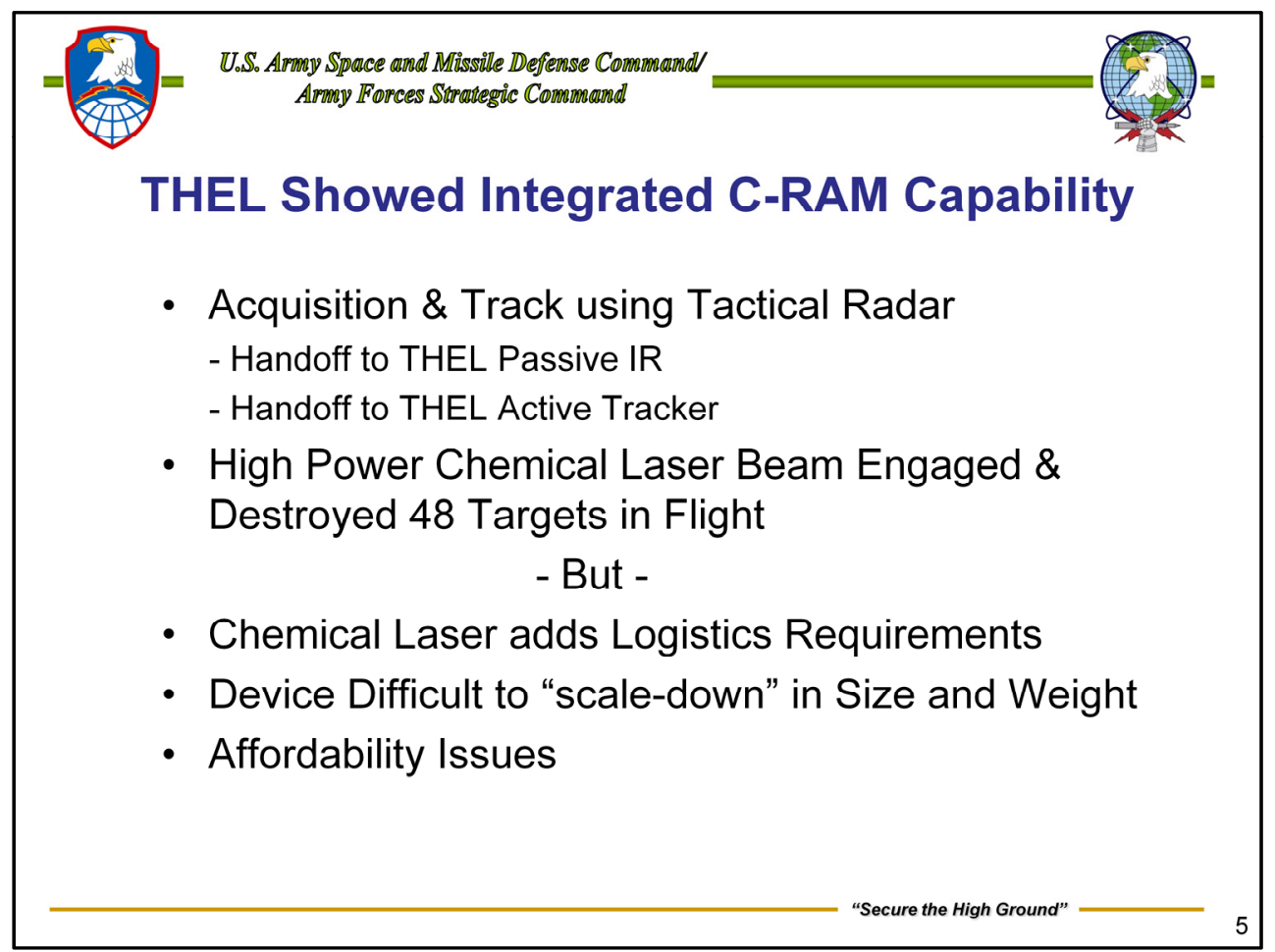

The THEL program, run from 1996 until 2005, successfully showed integrated CRAM capability with a high energy laser system using a deuterium fluroride chemical laser. As demonstrated on THEL, a radar track from different types of radars can be handed off to a laser weapon system's on board optical sensors. The THEL active tracker was able to establish a "laser quality track" of sufficient accuracy for the pointer tracker subsystem to direct the high power chemical laser beam on a total of 48 targets in flight and destroy them. Nearly all targets engaged with the THEL beam were destroyed, demonstrating a very high probability of kill.

But, concerns about logistics requirements and the difficulty of scaling down the size and weight of the chemical laser system contributed to the termination of the program in 2005. In addition, the Israeli government, which had been providing significant funding to the program, decreased their financial support in 2004. Furthermore, competing priorities in the Army's budget resulted in the elimination of additional investment in DF chemical laser technologies by the US Army, with a shift toward solid state laser technology development. 


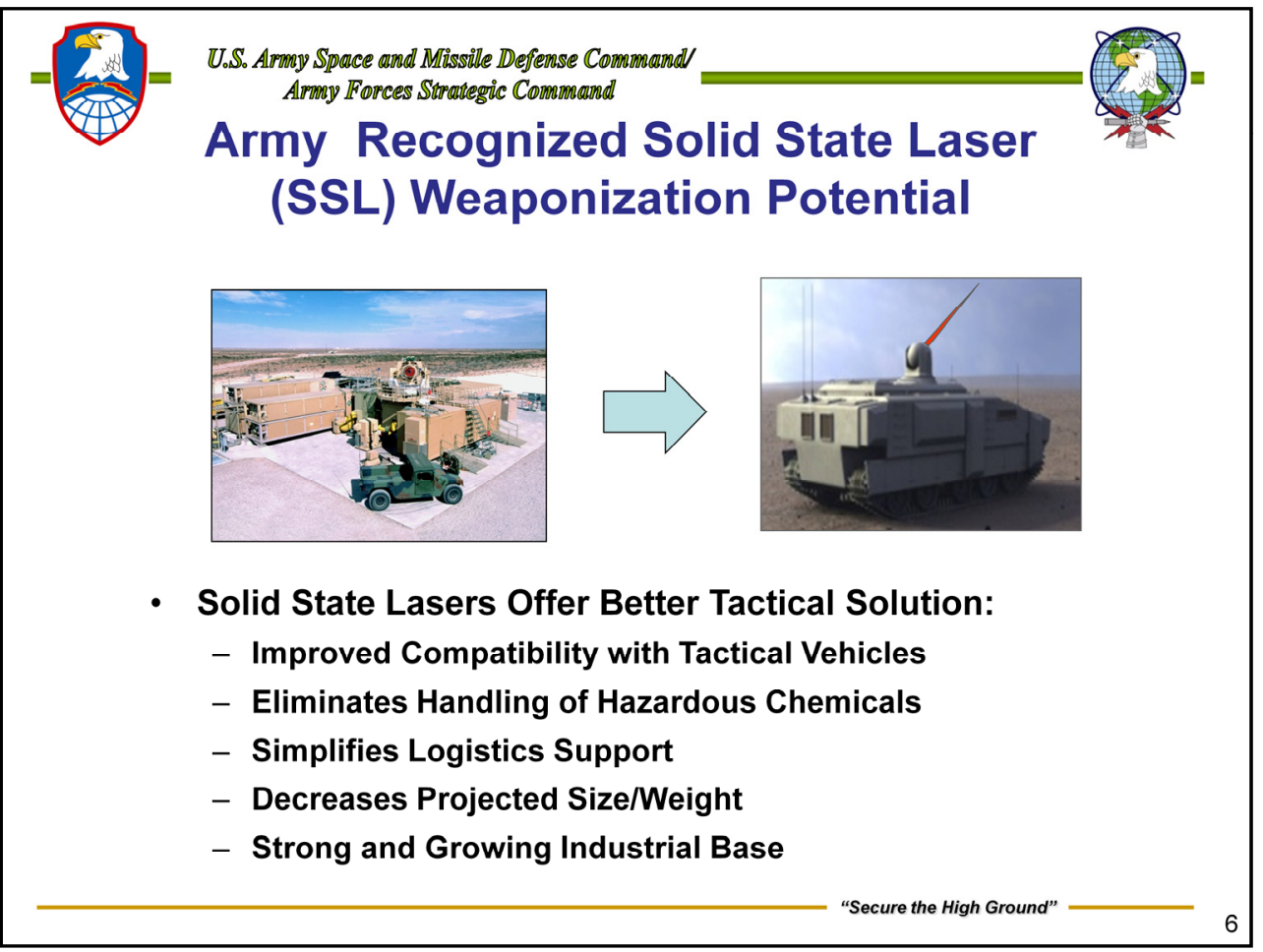

THEL successfully demonstrated C-RAM capability using mature chemical laser technology, but for a number of reasons the US Army decided in 2005 that solid state lasers offered a better solution to evolving mission requirements than chemical lasers.

Even though solid state lasers were the first type of lasers to be invented in the early 1960's, thermal management issues with flash lamp pumped SSL technology resulted in very slow increase in laser output power until the late 1990's with the development of diode pumped SSL devices. The power output of diode pumped SSLs has dramatically improved ( two orders of magnitude) over the last ten years and continues to improve with focused investment by the High Energy Laser Joint Technology Office and the services.

Solid State Lasers also offer all electric operation, eliminating the need to handle hazardous chemicals required by chemical laser systems. SSLs are expected to greatly simplify logistics support requirements compared to chemical lasers, with the main consumable being diesel fuel already in the inventory. SSLs are also expected to be more compatible with tactical vehicles with decreased size and weight relative to chemical lasers. In addition, a very strong and growing industrial base for SSL devices is helping to lower the costs of producing these devices. 


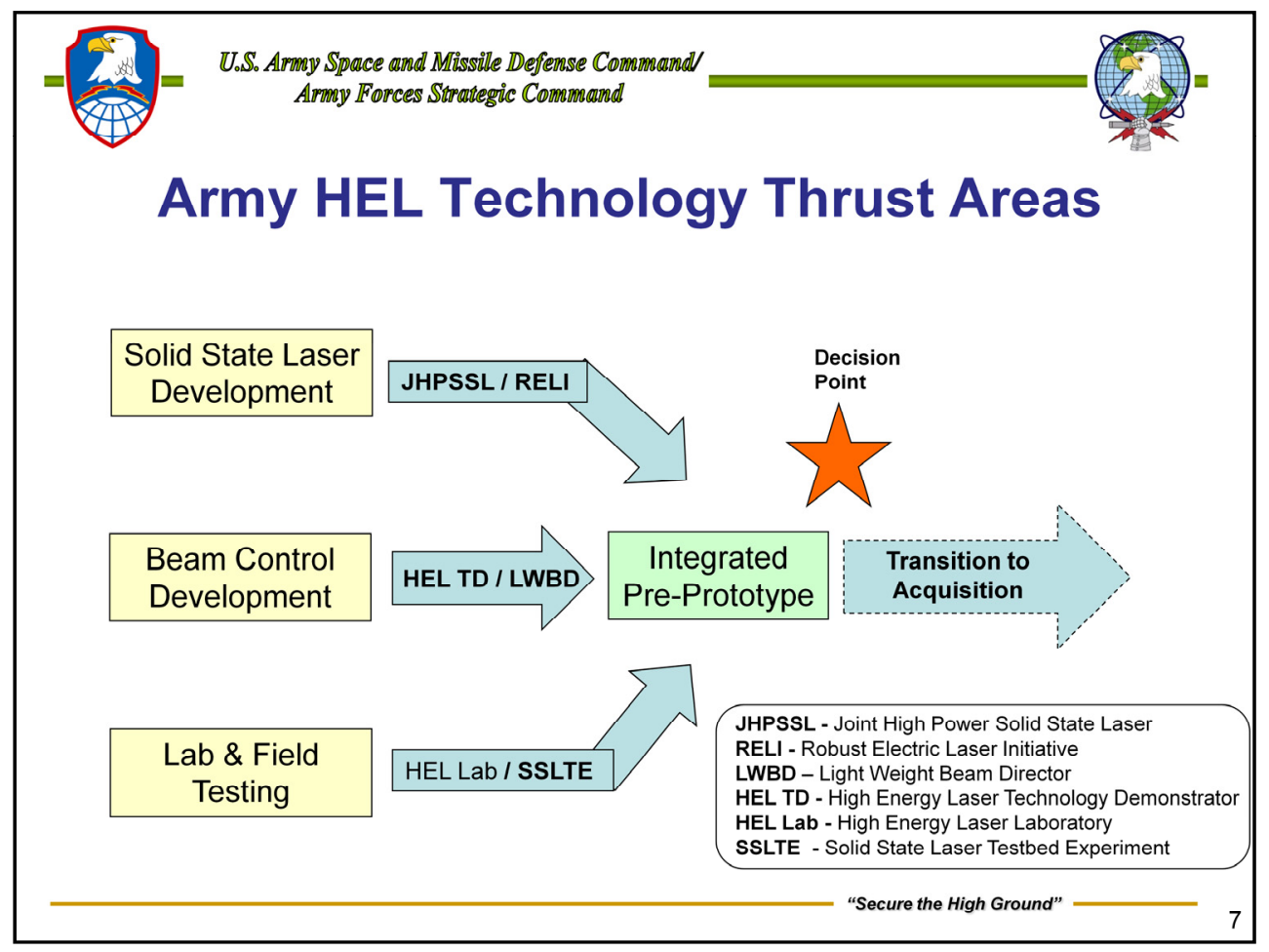

The current US Army High Energy Laser (HEL) Technology program has these three thrust areas; 1) Solid State Laser (SSL) Development, 2) Beam Control System (BCS) Development, and 3) Laboratory and Field Testing.

The first thrust area has successfully completed the Joint High Power Solid State Laser (JHPSSL) program, jointly funded by the HEL JTO and the services. The JHPSSL program successfully increased the power of solid state lasers from approximately $1 \mathrm{~kW}$ to $100 \mathrm{~kW}$ 's. The follow-on effort is called the Robust Electric Laser Initiative (RELI), which is focused on developing the next generation of SSL technology that is more compatible with tactical platforms, including ground, air, and sea based applications.

The BCS thrust is focused on integrating a ruggedized beam control system on a Heavy Extended Mobility Tactical Truck (HEMTT) and conducting low power testing against RAM targets under the HEL Technology Demonstrator (HELTD) program. The Light Weight Beam Director (LWBD) program is pursuing the next generation of BCS that will improve compatibility with tactical platforms and reduce cost by improved fabrication methods using advanced materials.

The Laboratory and Field Testing thrust utilizes an existing AMCOM facility to do in-house research supporting development of atmospheric compensation and field measurement techniques. In addition, full weapon power testing will be conducted using the Solid State Laser Testbed Experiment (SSLTE) currently under development at the High Energy Laser Systems Test Facility (HELSTF) on White Sands Missile Range, NM.

These thrust areas will lead to the development of an integrated pre-prototype system capable of demonstrating the functions of an HEL weapon system. The performance of the pre-prototype system will support the decision to transition SSL technology to an acquisition program within the Program Executive Office for Missiles and Space, which now has the mission of C-RAM and C-UAV. 


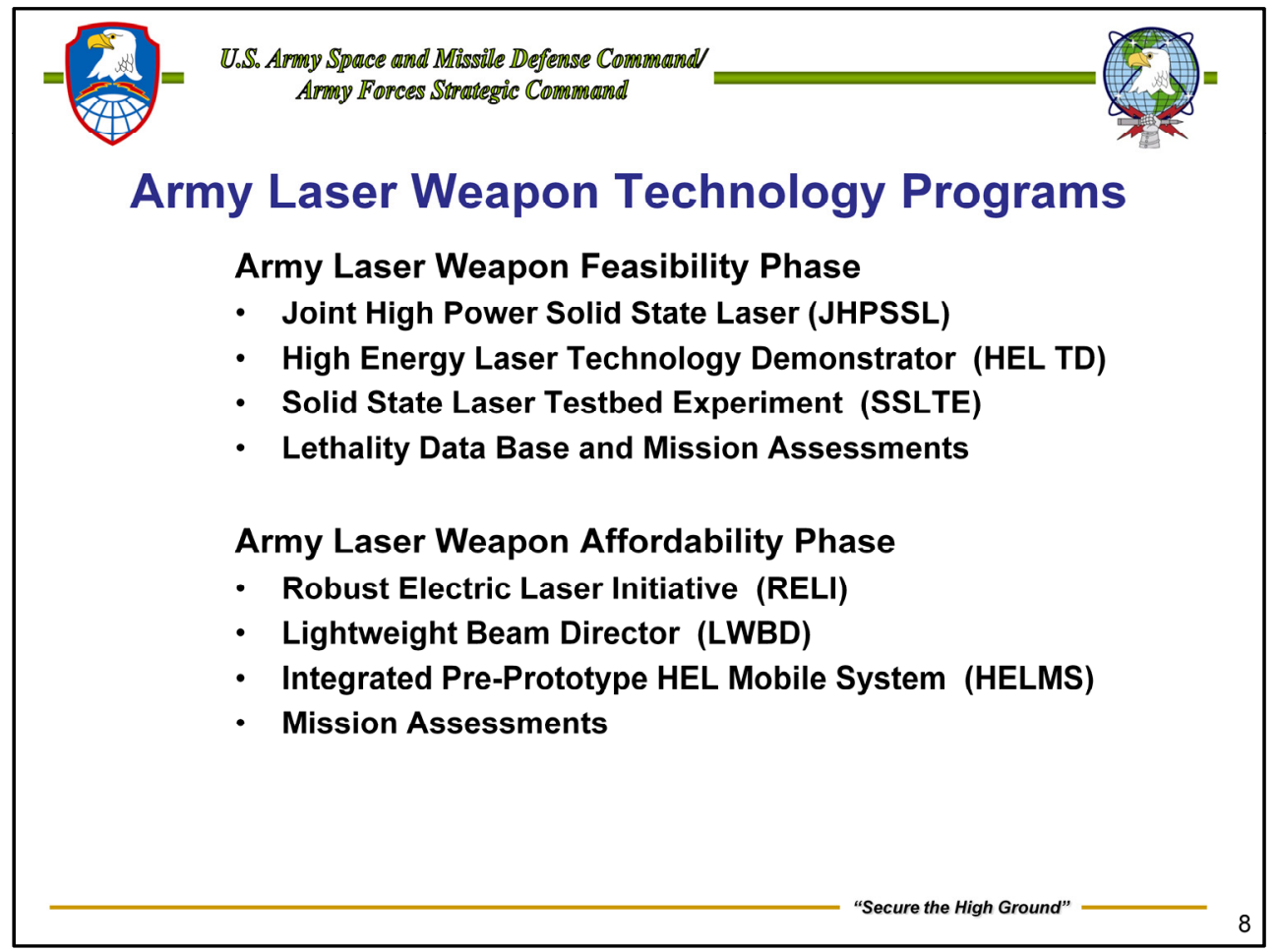

These are the major elements of the Army Laser Weapon Feasibility and Affordability Phases. In many respects, the feasibility phase is nearing completion with the demonstration of weapon class performance in the laboratory of the JHPSSL devices. In addition, the HEL TD program is expected to complete integration of a beam control system on a tactical truck and demonstrate low power testing in late 2011. The SSLTE is expected to be operational at weapon power levels by early next calendar year to support further development of the lethality data base at solid state laser wavelengths and mission assessments of the evolving capabilities of SSL's. I

The weapon affordability Phase elements include the RELI program to further advance the state-of-the-art (SOA) of SSL technology through improved efficiency and beam quality. The LWBD program provides the next generation of beam director to improve compatibility with tactical vehicles. The HELMS program integrates all the functional elements of a HEL weapon system in a "pre-prototype" configuration, incrementally improving performance as the SSL SOA continues to mature. 


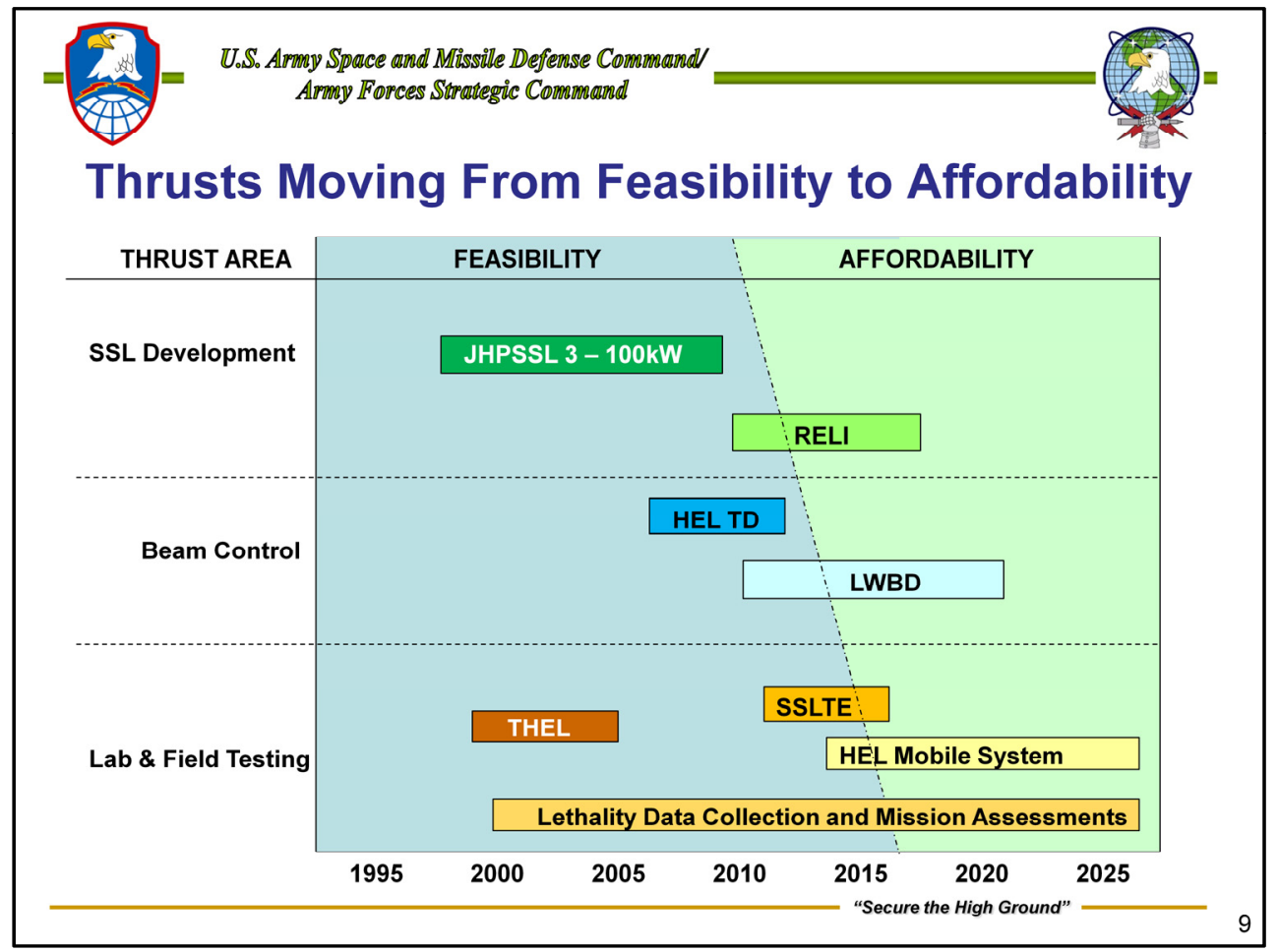

This chart provides a graphical representation of the relationship between the Army HEL technology program thrust areas as they evolve over time from demonstrating feasibility to demonstrating affordability in more tactical configurations. 


\section{Laser Weapon System Feasibility Programs}

$\checkmark$ JHPSSL - Demonstrate 100kW SSL in Laboratory

- HEL TD - Demonstrate SSL Beam Control at HELSTF

- SSLTE - Provide Capability to:

- Collect Lethality Data

- Perform Mission Assessments of SSLs

- Develop Tactics, Techniques, and Procedures

- Address Clearance to Fire 


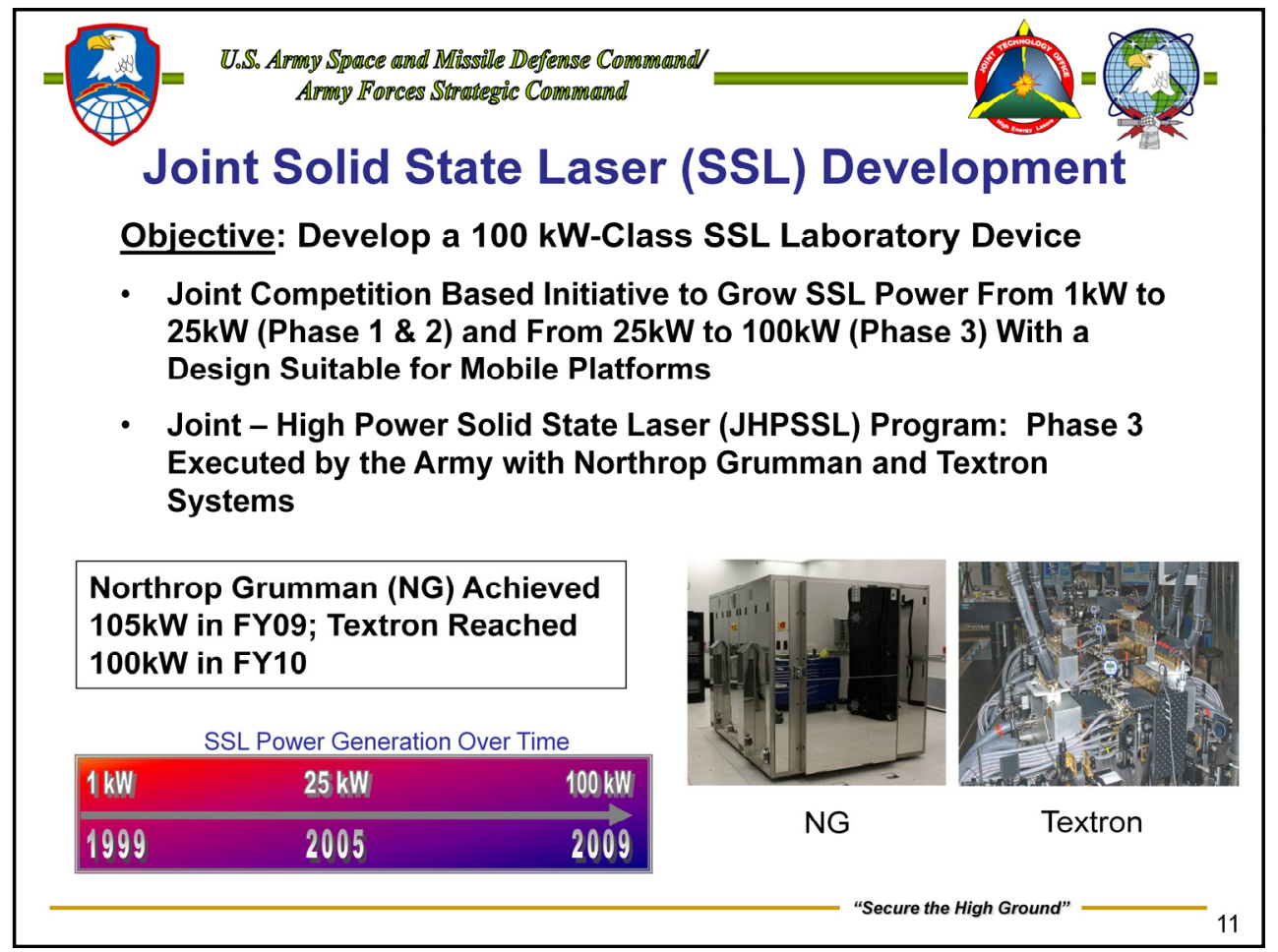

The Army developed, in cooperation with the High Energy Laser Joint Technology Office (HEL JTO), the Air Force, and the Navy, high average power solid state lasers. The purpose of the Joint High Power Solid State Laser (J-HPSSL) program was to develop solid state laser technologies with performance and packaging capabilities suitable for integration as elements of future tactical HEL weapon systems. The Phase $2 \mathrm{~J}-\mathrm{HPSSL}$ program goal was to demonstrate a laser with an average power of greater than $25 \mathrm{KW}$, high beam quality, long runtime, short start-up time, high wall-plug efficiency, high output power-to-mass ratio, and low beam jitter. This phase concluded with independent government testing at multiple locations at the end of FY05.

The Phase 3 program objective was to develop a 100kW-class, diode-pumped solidstate laser laboratory device with excellent beam quality relative to the diffraction limit (DL). Emphasis was placed on laser architectures that the government believed was favorable in terms of size, weight, efficiency, reliability, maintainability, supportability, environmental acceptability, and ruggedness.

The most important performance JHPSSL Ph 3 parameters were defined to be average output power, beam quality, electro-optical efficiency, and run time.

- Average power: The solid state laser average output beam power to be at least $100 \mathrm{~kW}$ at a wavelength that is highly transmissive through the atmosphere.

- Laser Beam Quality (BQ): The instantaneous laser beam quality threshold to be $2 \times \mathrm{DL}$ with a goal of $1.5 \times \mathrm{DL}$, based on a power-in-the-bucket (PITB) measurement in the far-field.

- Electro-Optical Efficiency: The threshold e-o efficiency value is $17 \%$ with a goal of $19 \%$. The e-o efficiency is the output laser power divided by the input electrical power provided to the laser diodes.

- Run Time: The laser device run time threshold to support the ability to maintain 5 second shots every 6.6 seconds (including start-up time) for 3 initial shots, followed by additional shots occurring on a Duty Cycle (DC) of $20 \%$, during a total time period of 200 seconds.

All of the above performance objectives have been demonstrated by direct physical measurements of the Phase 3 laboratory device except efficiency, which can be derived based on physical measurements and analysis. 


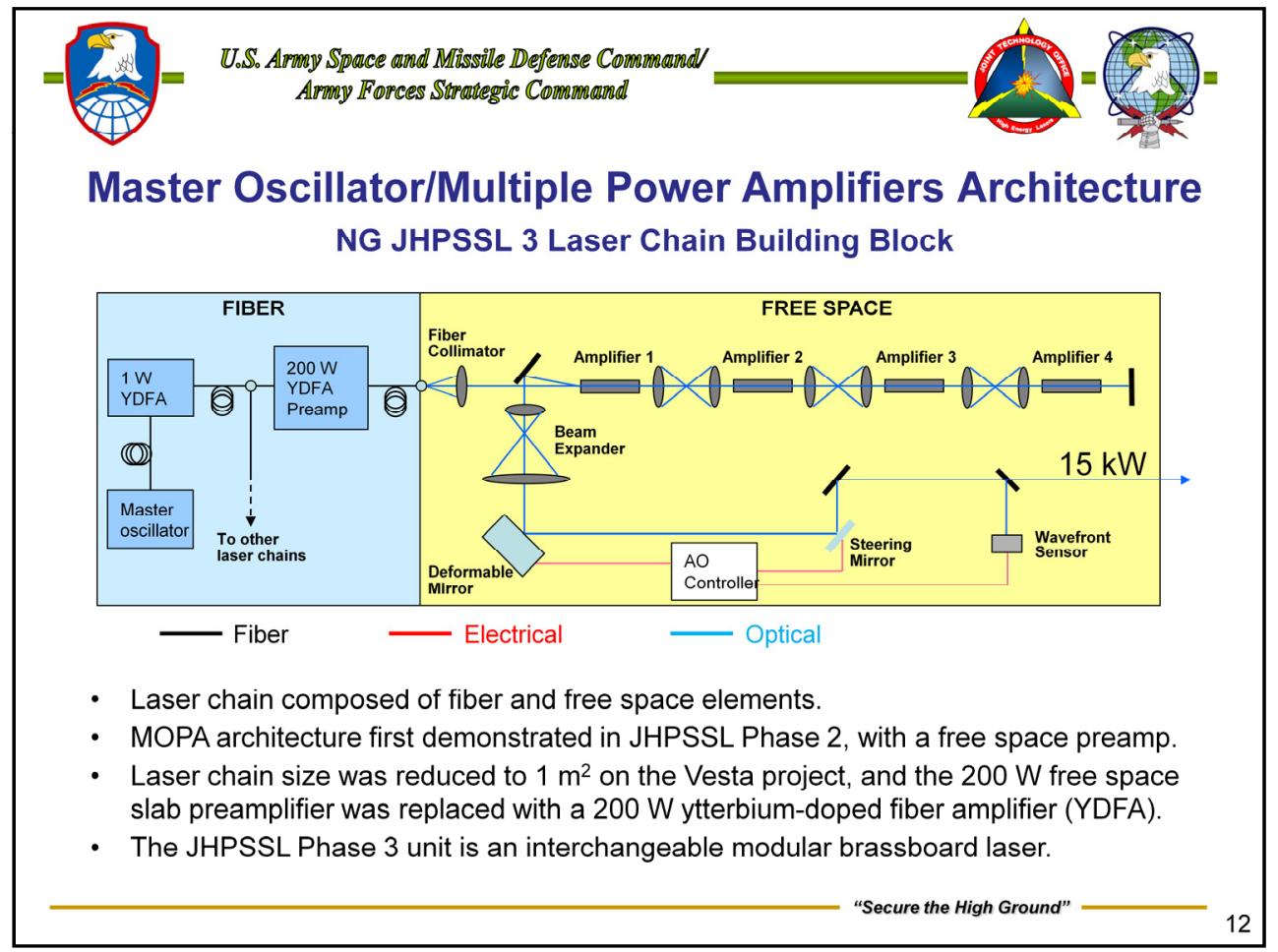

The next step in building a 100-kW laser is to incorporate four each of the gain modules into a laser chain. The laser chain has a hybrid fiber laser - free space design:

- Unlike the JHPSSL Phase 2 laser chain, which had a free-space preamplifier that used a slab gain module, the preamp for this generation was all fiber, taking advantage of the rapid advancement in high power fiber lasers that took place from 2003 to 2006.

- Also, in contrast to the JHPSSL2 laser chain, the size and weight were reduced significantly, to 1 $\mathrm{m}^{\wedge} 2$, and this is an interchangeable, modular brassboard laser.

- The blue section on the left is a schematic of the fiber front end:

- The beam starts in the master oscillator, which is then amplified by a commercially available $1 \mathrm{~W}$ ytterbium-doped fiber amplifier

- This 1W output beam is split multiple ways to seed a 200-W fiber preamplifier the 7 laser chains:

- This 200-W fiber preamp was developed in partnership with Nufern

- The fiber preamp produces a $200-\mathrm{W}$ output beam with $\mathrm{M}^{\wedge} 2<1.1$.

- The 200-W fiber output is collimated and sent into the free space portion of the laser chain

- The beam is shaped and makes a double pass of four 4-kW gain modules, resulting in a well saturated 15-16 kW output beam

- This high power beam then enters the beam conditioning back end section:

- A beam expander enlarges the beam and shapes it to fill the active area of a deformable mirror

- Because of the thermally induced OPD introduced by the gain modules, the 15-kW beam is aberrated and needs to be corrected

- Part of the beam is sampled and coupled into a wavefront sensor to provide feedback to the DM

- The resulting output beam has high output power coupled with excellent beam quality $(\sim 1.6 \times \mathrm{DL})$ 


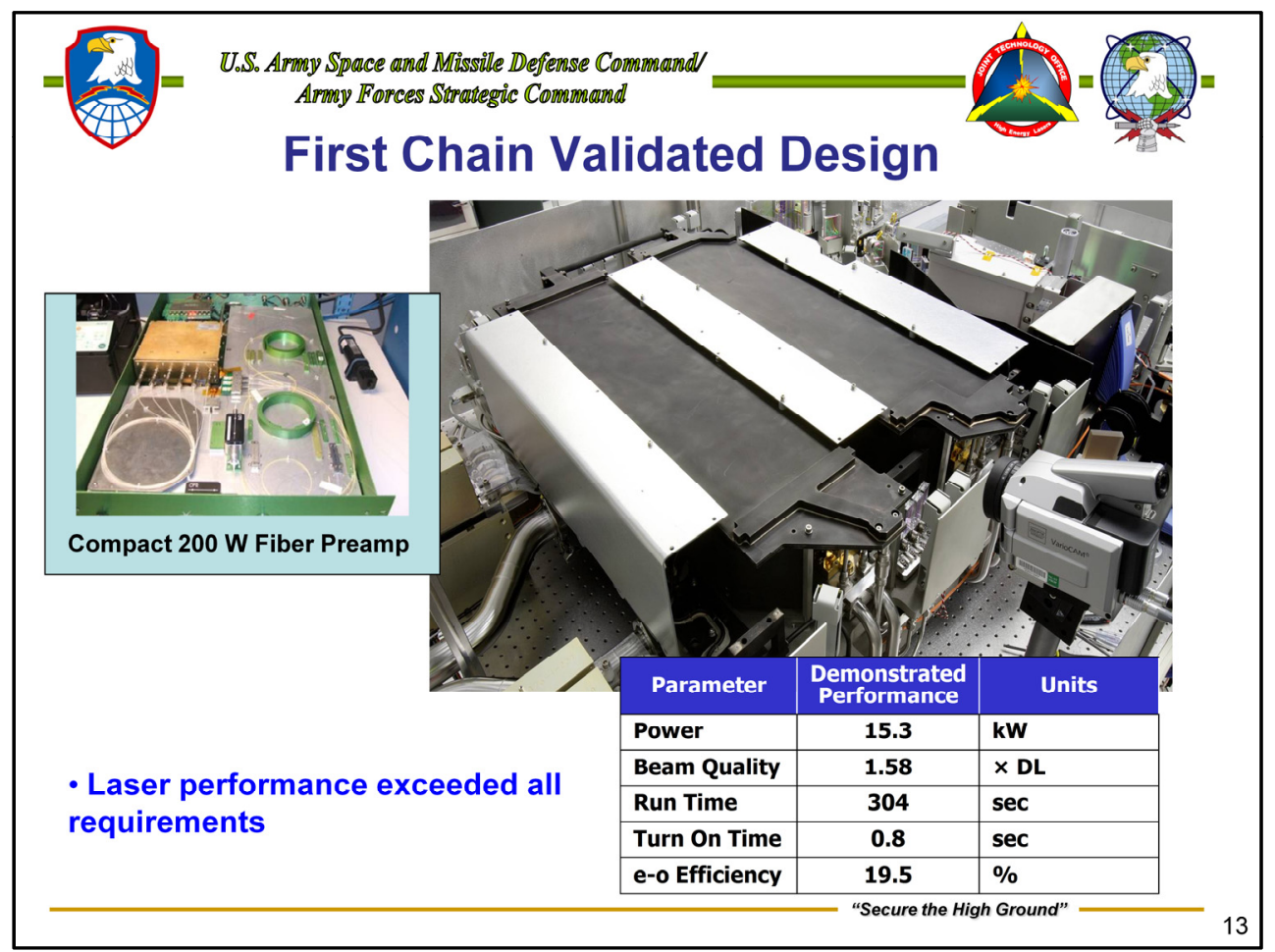

- This slide shows a image, on the right, of the first completed laser chain, and on the left, a picture of one of the $200-W$ fiber preamplifiers with its top removed

- The gain module section of the laser chain is under these black covers, and the beam conditioning section is at the back. The DM can be seen on the right.

- The first completed laser chain was demonstrated in late 2007:

- Performance parameters are shown in the chart:

-Output power was $15.3 \mathrm{~kW}$

-Beam quality was about 1.6 x DL (using vertical BQ definition)

-Run time was over 5 minutes

-Turn on time achieved the goal of less than 1 second

-E-O efficiency was about $20 \%$ 


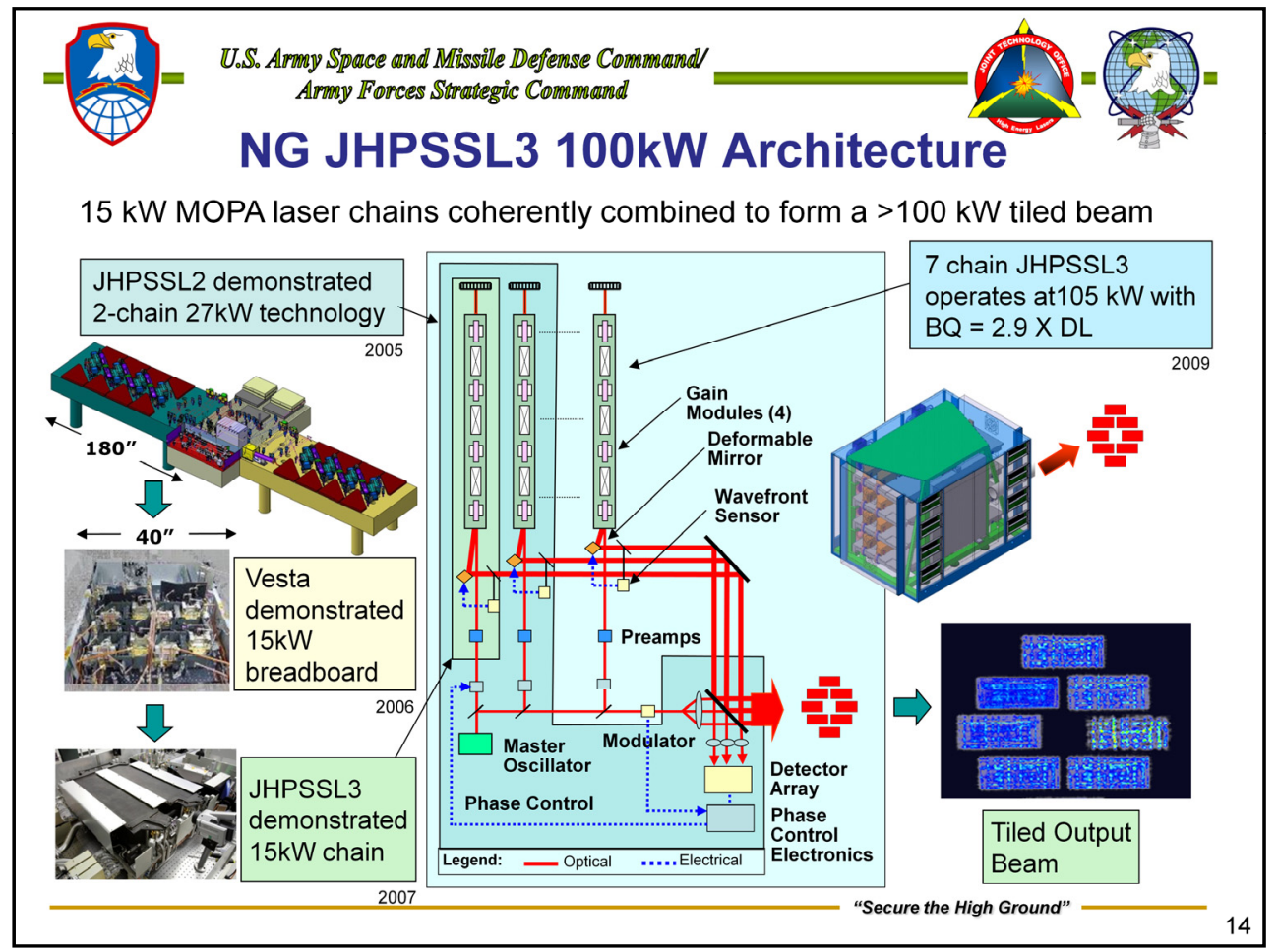

The 100-kW demonstration is the culmination of the Joint High Power Solid State Laser (JHPSSL) program. This program, which began in late 2002, consisted of three phases:

- Phase 1: Demonstration of enabling technologies that would make a 100-kW SS laser possible (high power optical coatings, high power gain modules, deformable mirror technology, etc)

- Phase 2: The goal of JHPSSL phase 2 was to demonstrate a 25-kW laser with good beam quality. Our JHPSSL2 solution was to coherently combine two $12-\mathrm{kW}$ laser chains. This was a laboratory demonstration, where each laser chain occupied a 180" long Newport bench, and the low power front end and high power back end occupying a $3^{\text {rd }}$ middle bench.

- In 2006, NG executed an internal R\&D project to produce the $2^{\text {nd }}$ generation laser chain, called "Vesta". This effort moved the high power laser chain from laboratory tabletop demonstration closer to the goal of an engineered, fieldable unit. The result of this effort was a laser chain which was substantially smaller and lightweight as compared to the JHPSSL2 laser chain, with it only measuring 40 inches on a side, or a $\sim 1 \mathrm{~m}^{\wedge} 2$ footprint. $2^{\text {nd }}$ generation gain modules were also developed for this laser chain. The Vesta chain put out $15 \mathrm{~kW}$, slightly more than the 10-12 kW put out by the JHPSSL2 chains, and had a demonstrated BQ of $1.3 \times$ DL.

- The final phase of JHPSSL, Phase 3 , began in 2006. Its goal was to build upon what was learned in the first two JHPSSL phases and demonstrate a 100-kW solid state laser with good beam quality. Shown at bottom left is the first completed JHPSSL3 laser chain, which was completed in 2007.

- In the middle is a schematic of the 100-kW laser system:

- The laser system is a MOPA system

- The beam begins at an MO with a $\sim 10 \mathrm{~mW}$ beam

- This beam is split 8 ways. Seven of the split beams go on to seed the seven high power laser chains

- The beam in each laser chain makes a double pass of four gain modules, resulting in $\sim 15 \mathrm{~kW}$ output power from each chain

- The seven beams are tiled side by side, as shown in this image on the right, in the beam combiner

$\circ$ The $8^{\text {th }}$ beam split off from the MO is frequency-shifted and provides the reference beam for determining the relative phases of each laser chain beamlet

- A phase control loop is used to phase all seven chains to produce a $100-\mathrm{kW}$ output beam that behaves like a sinale coherent beam. 


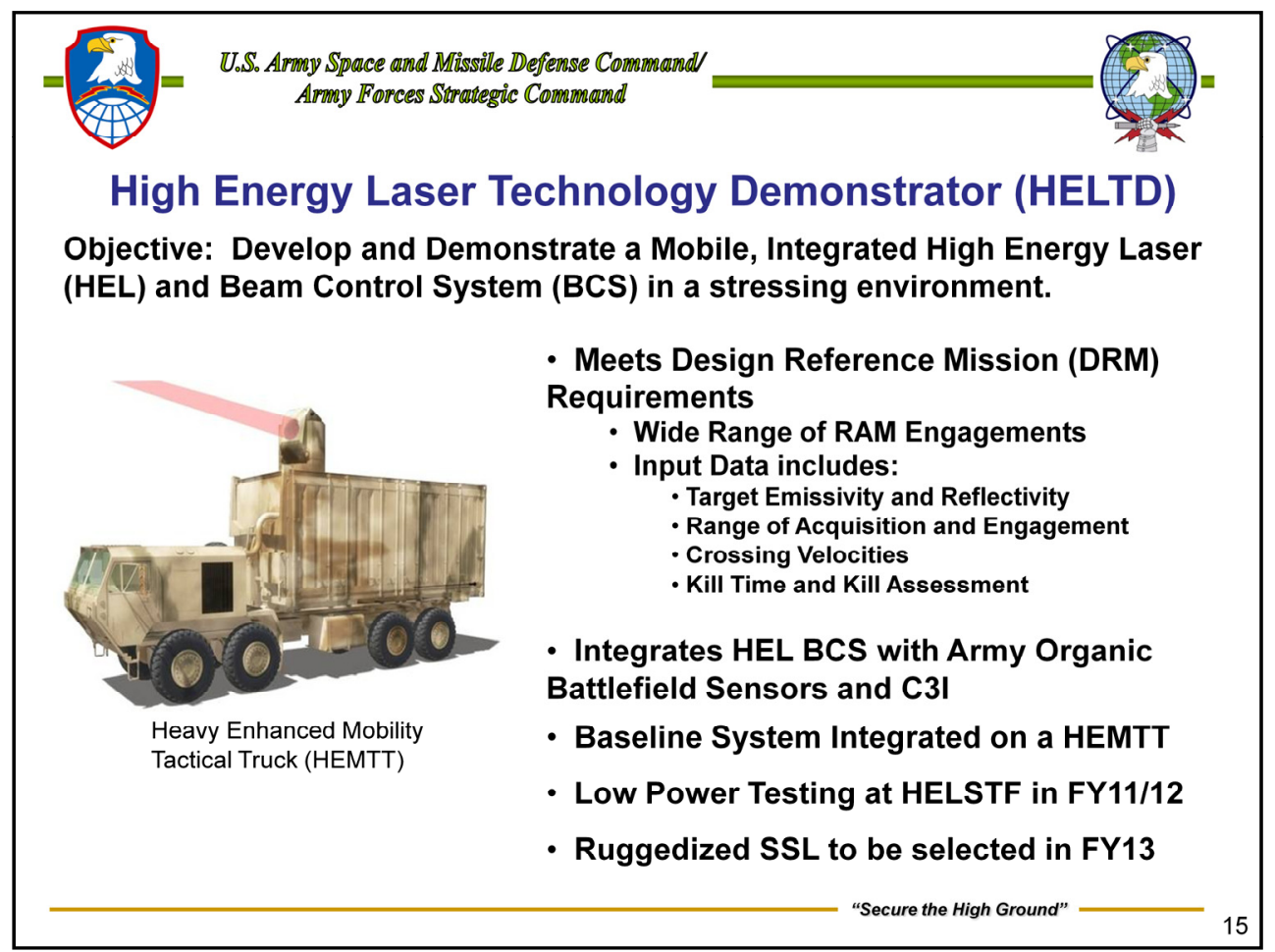

The High Energy Laser Technology Demonstrator (HEL TD) is an Army 6.3 funded effort that is developing a mobile solid state laser system to demonstrate full functionality of a SSL weapon system to Counter RAM in a relevant operational environment at HELSTF. The HEL TD is designed to have limited residual operational capability, with strong emphasis on minimal personnel requirements, packaging that allows limited field-level replacement, and access for maintenance. It will also be designed for battlefield integration, including C-17 transportability and $\mathrm{C} 3$ compatibility with current operations. The design is also modular to allow for future upgrades.

The HEL TD program is structured to first integrate a beam control subsystem (BCS) and low power laser on a Heavy Expanded Mobility Tactical Truck (HEMTT) to conduct acquisition and tracking tests at HELSTF in FY11 under SMDC contract with Boeing SVS, Albuquerque, NM. The HEL TD BCS will then be used in conjunction with the SSLTE high power device to demonstrate RAM shoot-down capability in FY12. The program will then initiate a competition to ruggedize a high power SSL for integration onto the HEMTT which will be available for testing in FY15-16. The prime power system will be on a towed trailer, while the thermal management system may be incorporated on the HEMTT or on the trailer. Additionally, BMC3I will be optimized for current operations. 


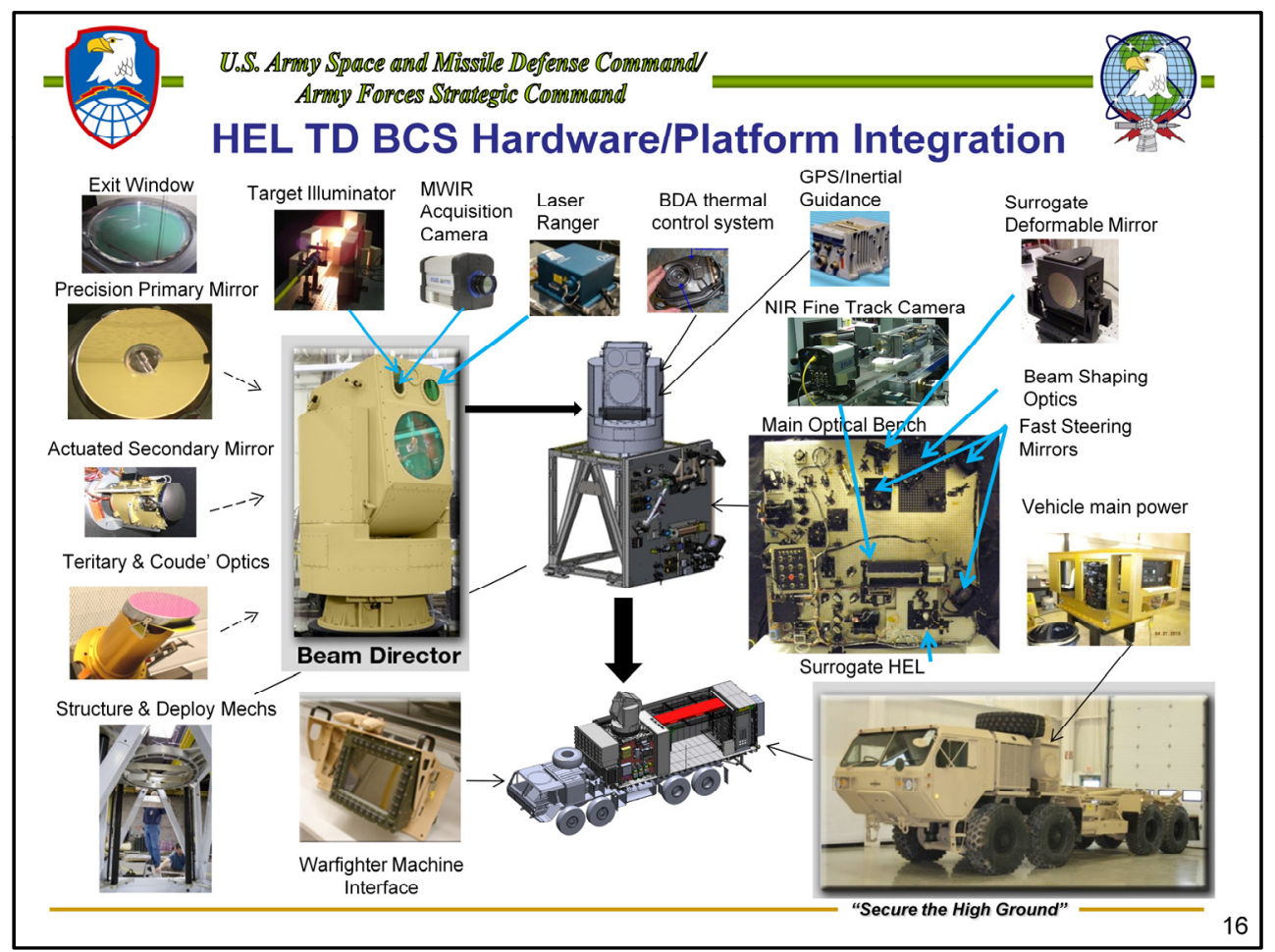

(center left) The $50 \mathrm{~cm}$ aperture Mersennes-type telescope was assembled and tested at Brashear showing better than 1/10 wave rms wavefront error. The az and el gimbals are capable of 120 degree/sec slew.

(far left) The output window is Lithosil with an AR coating, The primary mirror is zerodur on a 9 point mount incorporating a tuned mass damper.. The secondary, tertiary, and coude optics are single crystal silicon with ion sputtered coatings

(10 o'clock) The Target Illuminator Laser (TILL) is a $300+$ Watt (1550 nm wavelength) IPG fiber laser.

(11 o'clock) FLIR 6700 MWIR camera (InGAs) with Stingray zoom lens for passive acquisition and captures return from 1.55 micron TILL for transition to active acquisition

(12 o'clock, left) The Laser Range Finder provides distance to target for fine tracker and beam director focus settings for CRAM engagement from 300 meters to $9 \mathrm{~km}$.

(12 o'clock, right) A $300 \mathrm{~W}$ chiller loop fin the beam director above the gimbals provides precise thermal control to stabilize operational performance of tracker components. Small compressor is shown.

(1 o'clock) HEL TD's embedded GPS/Inertial Navigation assembly (Honeywell 74G) is located close to tracker.

A second back-up unit is located in the BDA support structure.

(2 o'clock, outside) Low power testing will be performed with a flat mirror in place of a Deformable mirror being developed under a JTO sponsored adaptive optics effort.

(center right) The main optical bench includes auto-alignment sources and sensors using information from the incoming laser, the telescope structure, and the target image. Three Fast steering Mirrors (Single Crystal Silicon ) provide correction .Gain and phase margins at the design goal of $400 \mathrm{~Hz}$ have been demonstrated. Capable of up to $3 \mathrm{kHz}$. Beam Shaping objects provided coring and clipping for the HEL beam to be selected. Low Power testing will use an IPG150W surrogate HEL mounted on the lower RHS of the bench.

(4\&5 o'clock) Standard diesel generator provides all power for HEL TD through low power test. The HEMTT A4 is the platform for HELTD. Made by Oshkosh.

(7 o'clock) The operator's display for HEL TD is a standard hardened Warfighter Machine Interface (WMI) display based on a standard air defense and Avenger C3I interfaces to support soldier familiarity and interoperability of the system. HEL TD has manual as well as automatic modes.

(8 o'clock) The robust steel structure provides rigidity and damping necessary to control jitter to the beam director from its mount. 


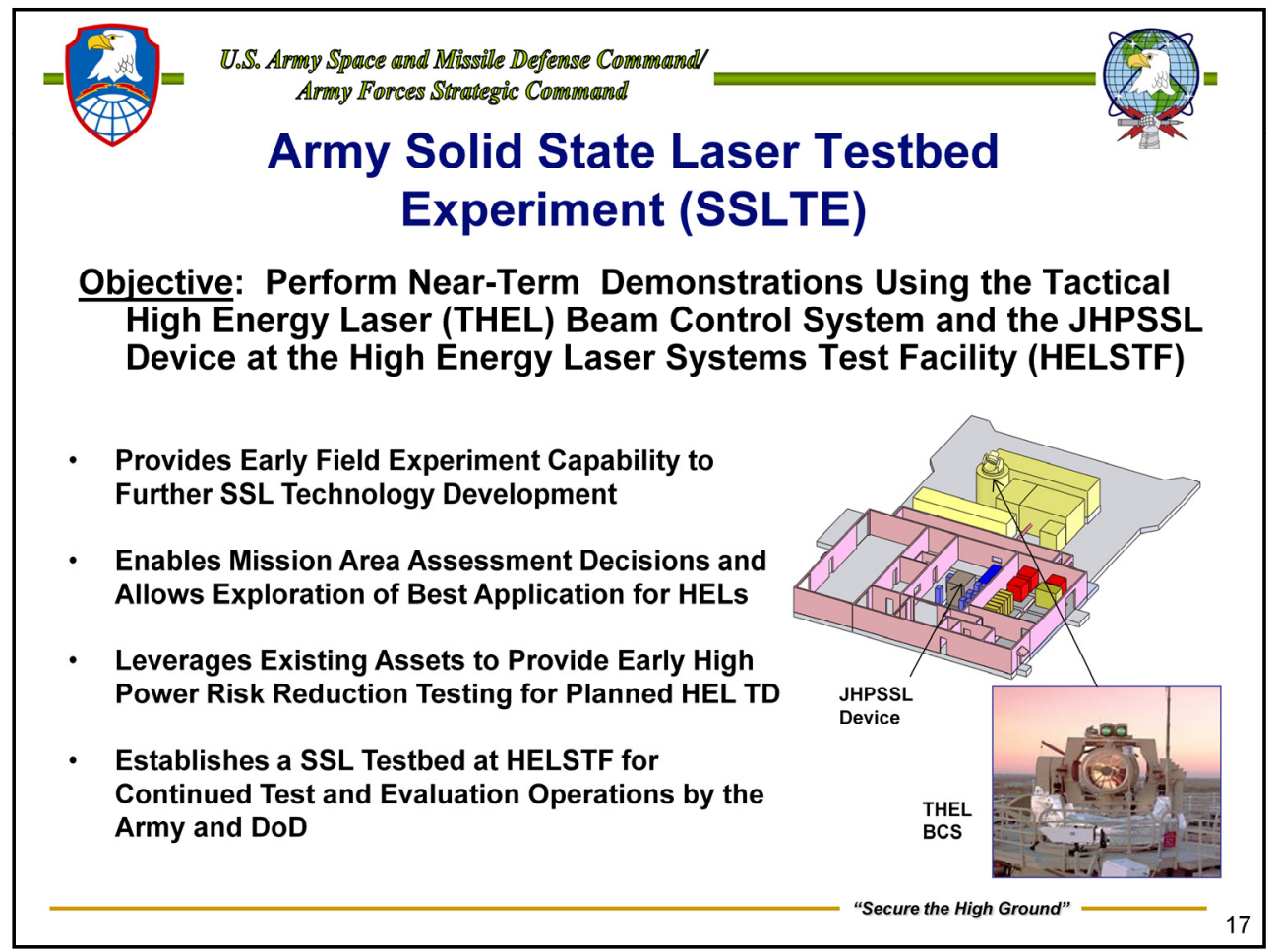

The solid state laser test bed (SSLTE) will provide unprecedented capability to conduct atmospheric propagation experiments, command and control experiments, and lethality testing - both dynamic and static. The SSLTE will enable the US Army to make mission area assessment decisions to determine the best mission applications for HEL weapon systems.

The SSLTE leverages existing assets, including the JHPSSL 100kW laser, the THEL beam control subsystem, and the command, control, and communication (C3) subsystem to minimize costs. The elements required to design and develop an enclosure for the laser subsystem and the interface to the beam control system and C3 subsystem are shown here. The environmental enclosure is essential for providing the environment (cleanliness, humidity, temperature, and vibration control) required by the laser subsystem to meet the required performance level.

The environmental enclosure will provide the interface between the laser subsystem and commercial/battery pack electrical power, closed-loop cooling assembly, and HVAC. It will also include the Control Room with links to operate and test the laser independent of the beam control system and C3 subsystem 


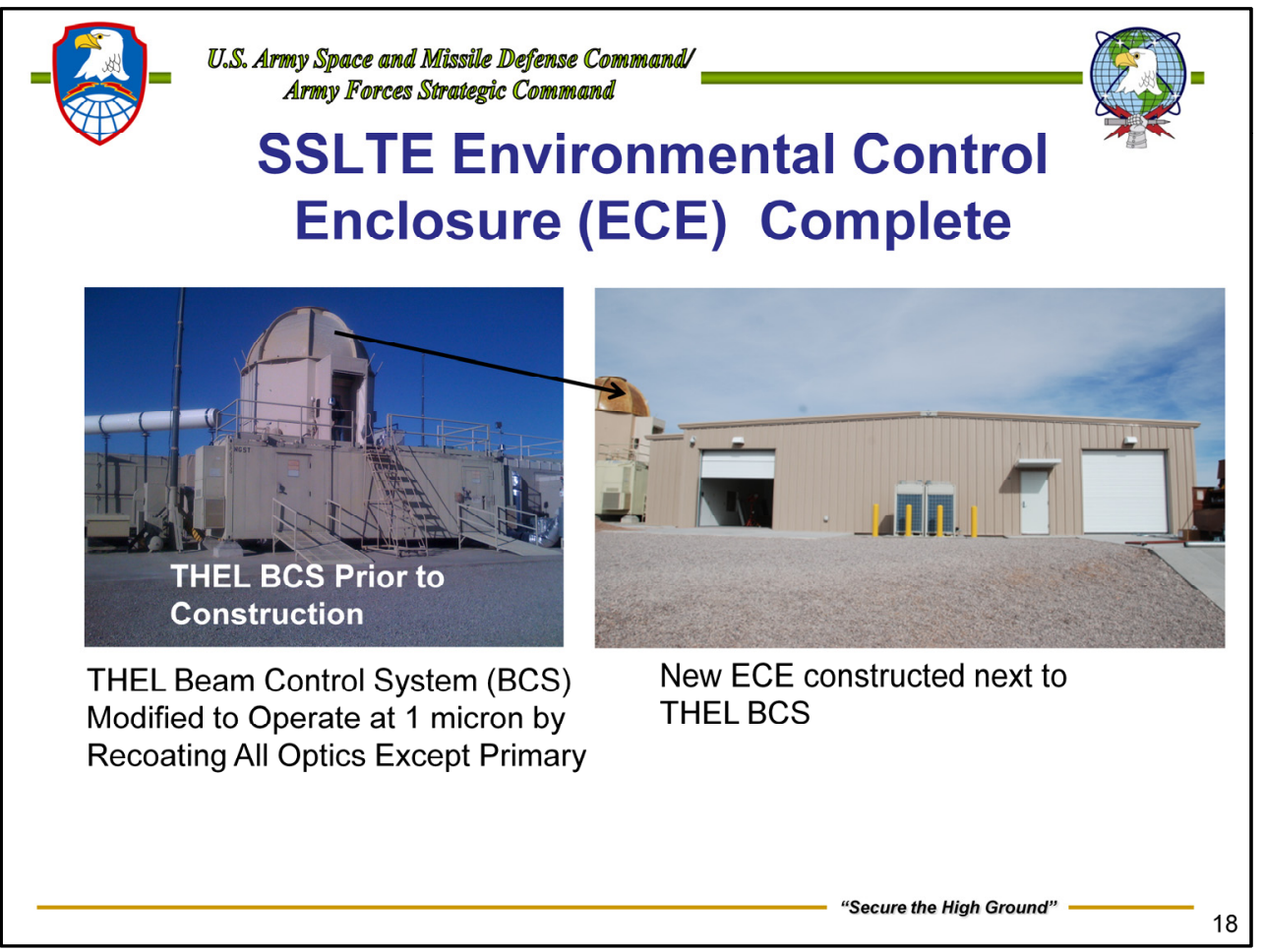

Current schedule completes the construction by late January 2011, with the JHPSSL 100 kW being shipped from Northrop Grumman in Mar 2011 and installed in the ECE by Jun 2011. The Basic Test Program is scheduled to begin in Jul 2011 and completed by Oct 2011. Continued lethality testing and demonstrations are planned through FY 2014. Future sustainment of the facility beyond FY2014 is anticipated as other service customers desire to conduct risk reduction and lethality testing for their specific missions and HEL programs. Vulnerability of conventional weapons to laser illumination is also anticipated to be an area of increasing customer interests. 


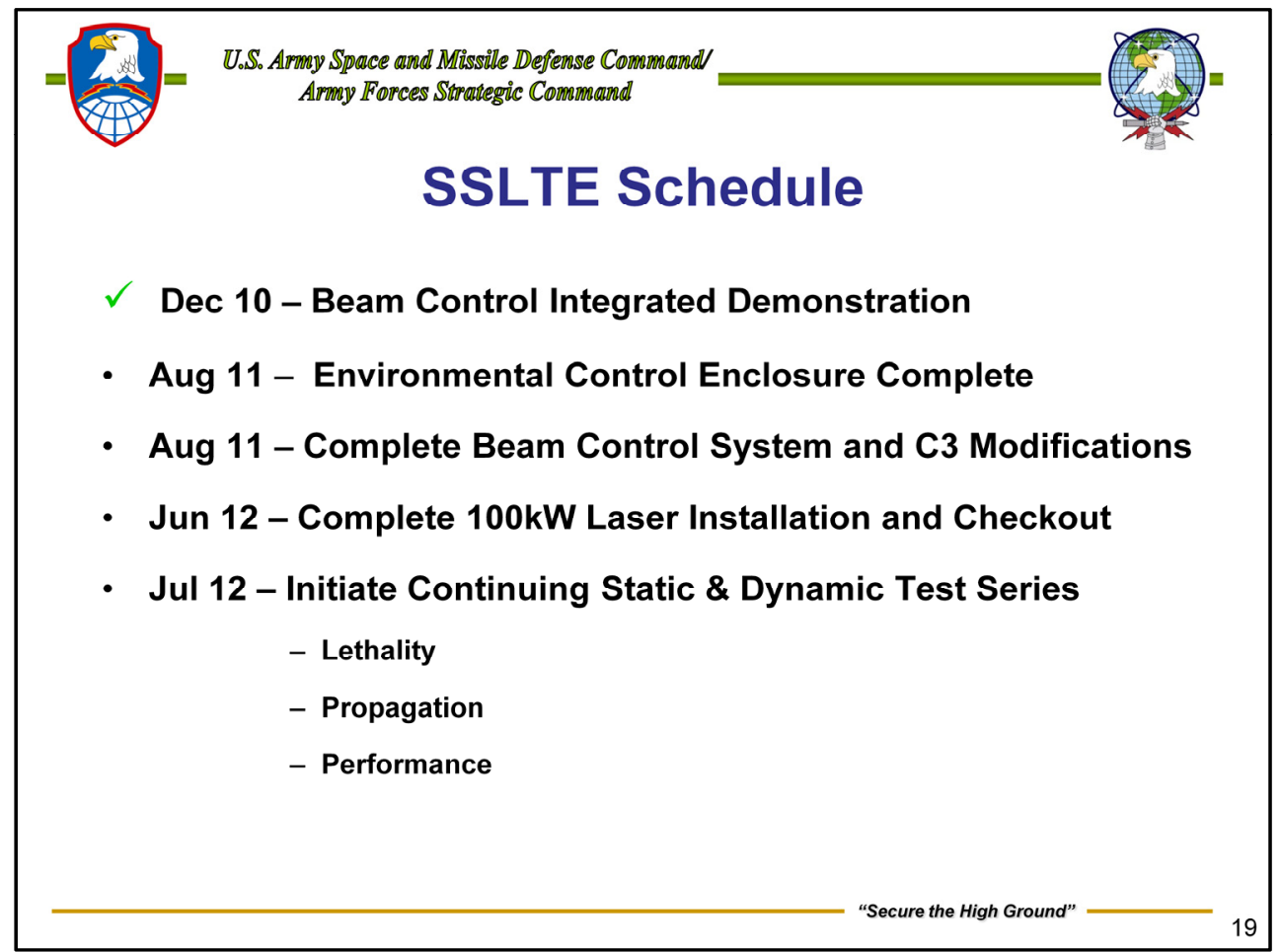

The current schedule completes the ECE construction by late August 2011, with the JHPSSL $100 \mathrm{~kW}$ being shipped from Northrop Grumman to the High Energy Laser Systems Test Facility (HELSTF) and installed in the ECE by Jun 2012. The test program is scheduled to begin in Jul 2012.

Continued lethality testing and demonstrations are planned through FY 2014. Future sustainment of the facility beyond FY2014 is anticipated as other service customers desire to conduct risk reduction and lethality testing for their specific missions and HEL programs. Vulnerability of conventional weapons to laser illumination is also anticipated to be an area of increasing customer interests. 


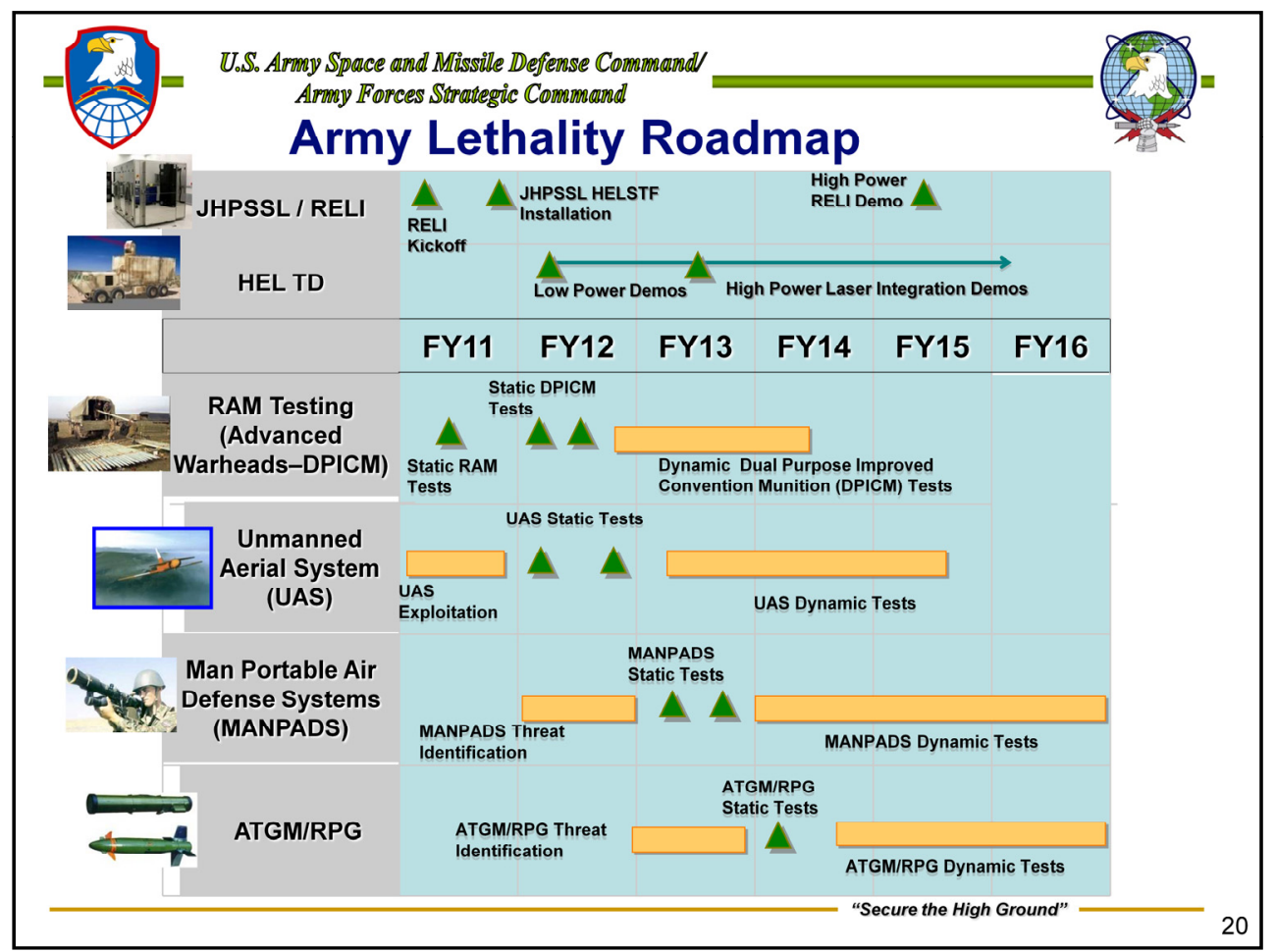

The Army's high energy laser development program is threat driven. A key element in developing any weapon system is understanding the physics and thermal dynamic effects of the target - laser interaction. One of the key requirements for a laser directed energy weapon is the amount of energy that must be deposited on a target to neutralize it.

This lethality roadmap displays a methodical approach in gaining the required understanding on a variety of threats that high power lasers can be used to negate or destroy. Lethality testing against this threat spectrum is ongoing.

When completed, the solid state laser test bed experiment will provide a weapon power, 1 micron wavelength, lethality testing capability to conduct the testing against the types of threats indicated on the Lethality Roadmap. 


\section{Laser Weapon System Affordability Programs}

- Robust Electric Laser Initiative +:

- Lockheed/Acculight ${ }^{\star}$ - Spectrally Beam Combined Fiber Lasers Using Multilayer Diffraction (MLD) Gratings

- Raytheon* - Planar Wave Guide (PWG) single beam Master Oscillator Power Amplifier (MOPA) laser

- Northrop Grumman** - Coherently Combined Fiber Lasers Using Active Phase Control

- General Atomics** - Thin Slab, Liquid Cooled, Power Oscillator

- Boeing* - Thin Disk

- Light Weight Beam Director Initiative:

- Brashear Built Telescope Structure, Refractive Optic Design

- High Energy Laser Mobile System

- Integrated Pre-Prototype System on a Tactical Vehicle

* US Army SMDC/ARSTRAT Managed

** US Air Force Research Laboratory Managed 


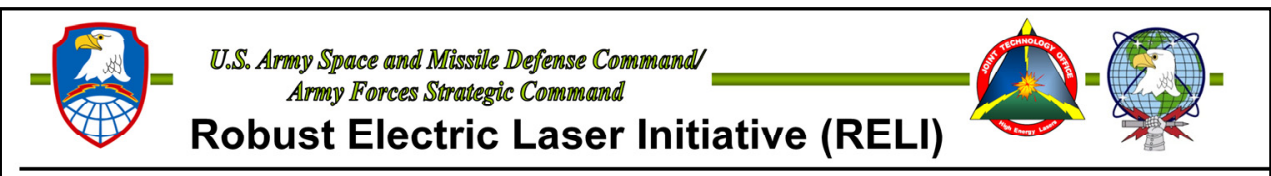

Objective: Develop 'robust, 'fieldable', and 'tailorable' Electric Laser Technology for transition into military HEL weapon systems

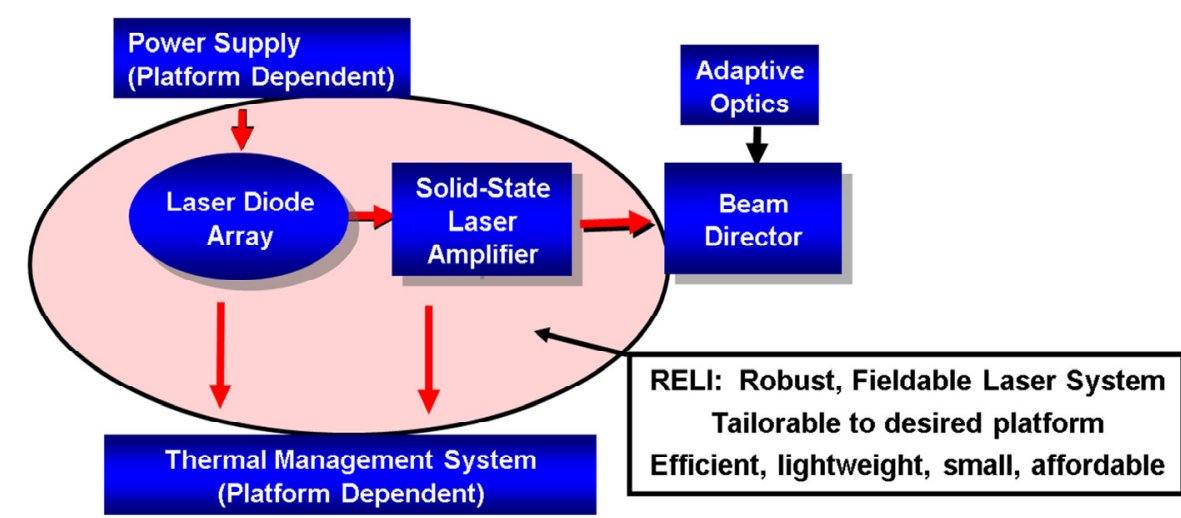

RELI will focus on laser efficiency and beam quality and minimizing weight and volume. It will not develop TMS or power production and conditioning technology.

"Secure the High Ground"

The objective of the joint Robust Electric Laser Initiative (RELI) is to develop robust, fieldable, and tailorable electric laser technology for transition into military HEL weapon systems.

The base contractual effort will culminate in an Interim Review (IR) for a $25 \mathrm{~kW}$ class laboratory demonstrator of the robust electric laser porposed and a Concept Design Review (CoDR) of a $100 \mathrm{~kW}$ class envisioned military laser system. The first option will be to build and test the $25 \mathrm{~kW}$ class electric laser in a laboratory.

Four contractors were awarded the base effort, each with a different approach to the meet the RELI requirements. The winners were Lockeed/ Acculight (Seattle, WA), Ratheon (El Segundo, CA), Northrop Grumman (Redondo Beach, CA) and Gereral Atomics (San Diego, CA). These contracts are being jointly funded by the Army, Air Force and the High Energy Laser Joint Technology Office. Later, another contractual effort was initiated with Boeing (Albuquerque, NM) to further their work on the thin disk laser. 


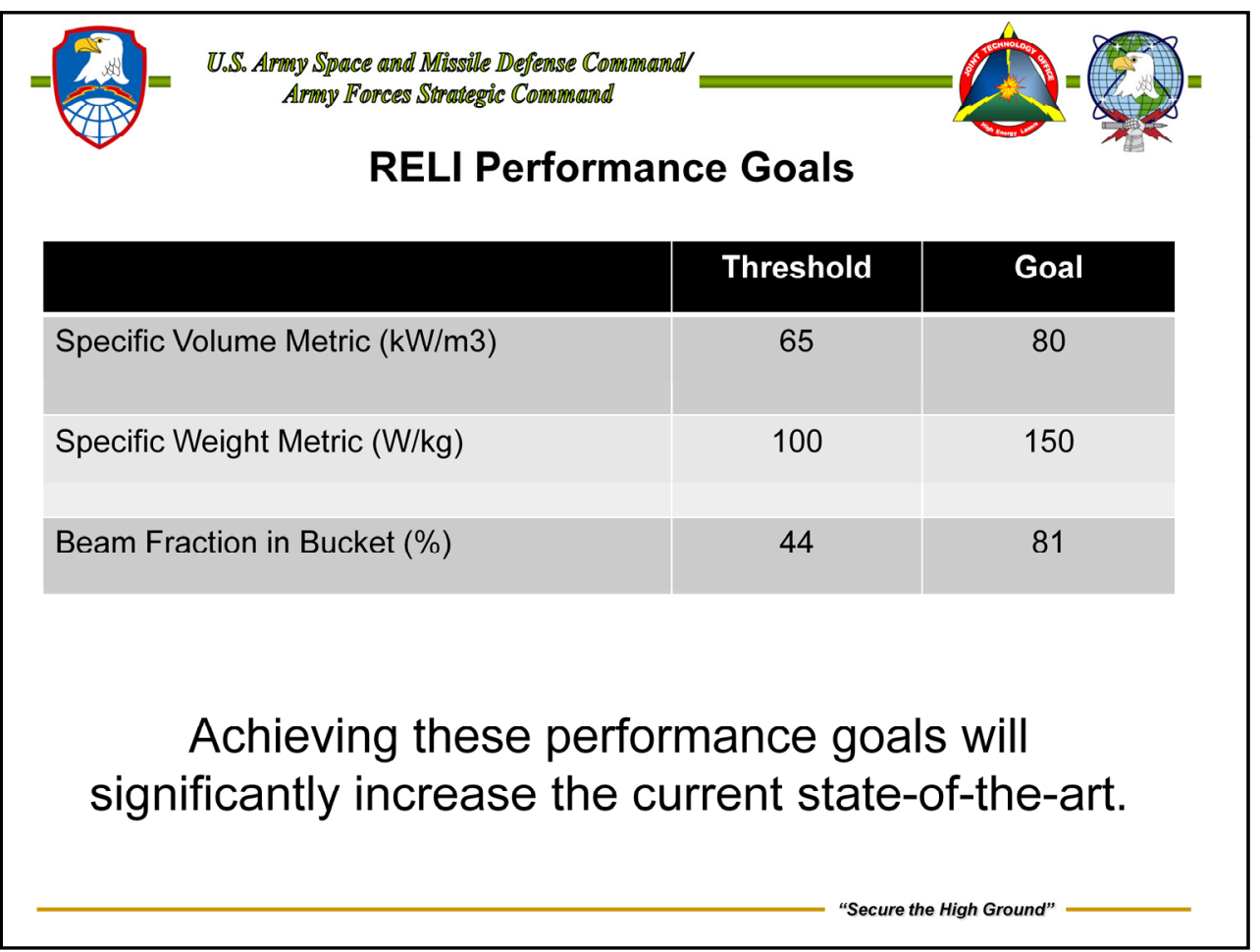

The RELI program is intended to advance the state-of-the-art of solid state lasers in several ways as indicated in the chart. If the specific volume and weight metrics for the RELI program goals are met, it will improve the SOA by roughly a factor of 10.

Another substantial improvement is the beam fraction in a far-field bucket, or in effect, energy on target. Compared to JHPSSL beam fraction of power in the bucket levels, a $\sim 4 \mathrm{X}$ improvement is anticipated for RELI.

Note: The DARPA HELLADS specific weight metric of $5 \mathrm{~kg} / \mathrm{kW}$ equates to 200 $\mathrm{W} / \mathrm{kg}$, much more stressing than the RELI specific weight metric goal of $150 \mathrm{~W} / \mathrm{kg}$. 


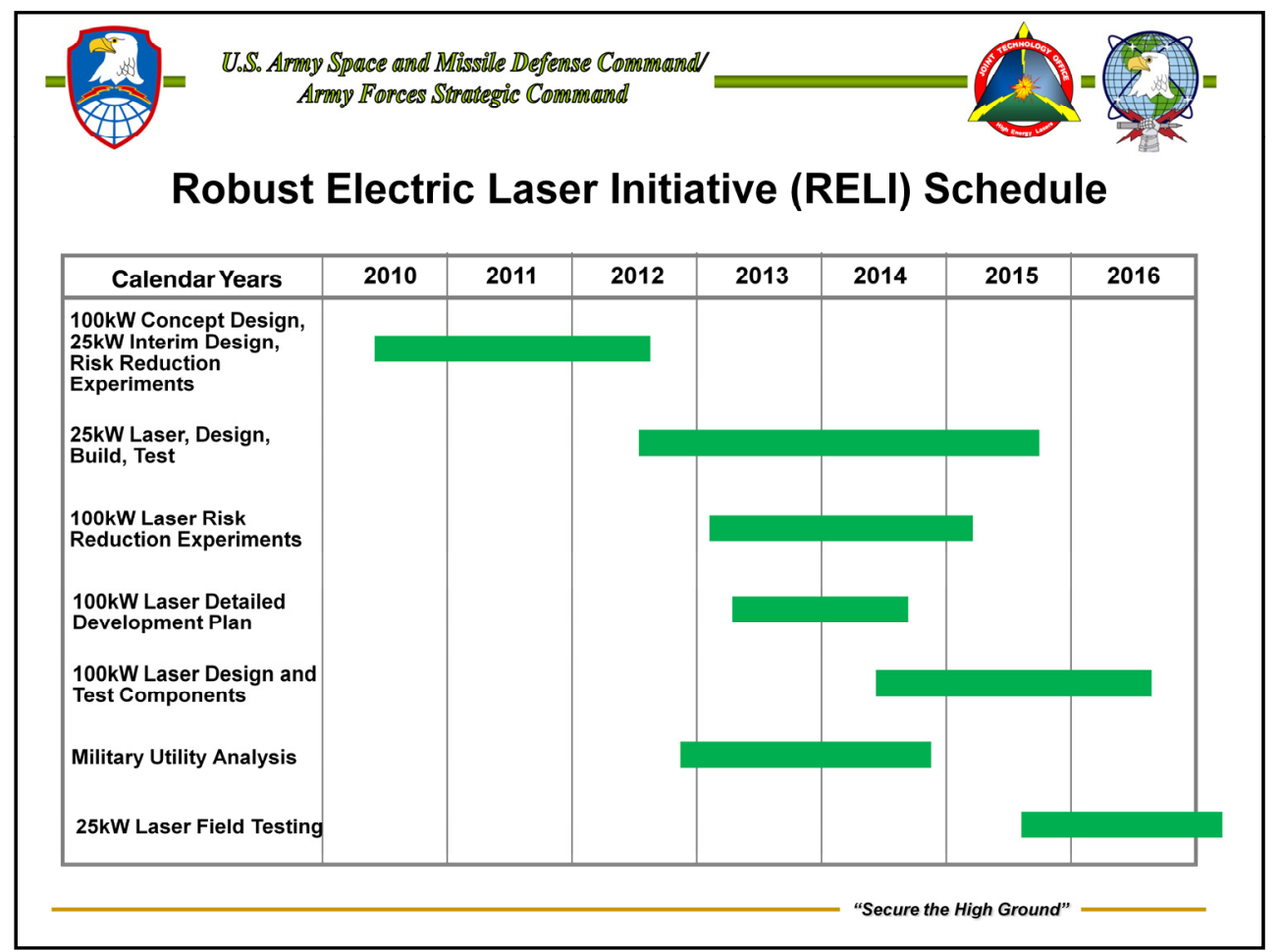

This chart represents a top level schedule for the RELI program. The four RELI contractors are currently working the first bar indicated, which ends in mid to late 2012. Two or more contractors are expected to be awarded the second activity indicated, i.e., the development and laboratory testing of a $25 \mathrm{~kW}$ system by 2015 . Other options on the RELI contracts are indicated and will be executed in accordance with the availability of funding.

The Boeing thin disk effort is on a timeline similar to RELI to modify an existing thin disk system to improve beam quality. 


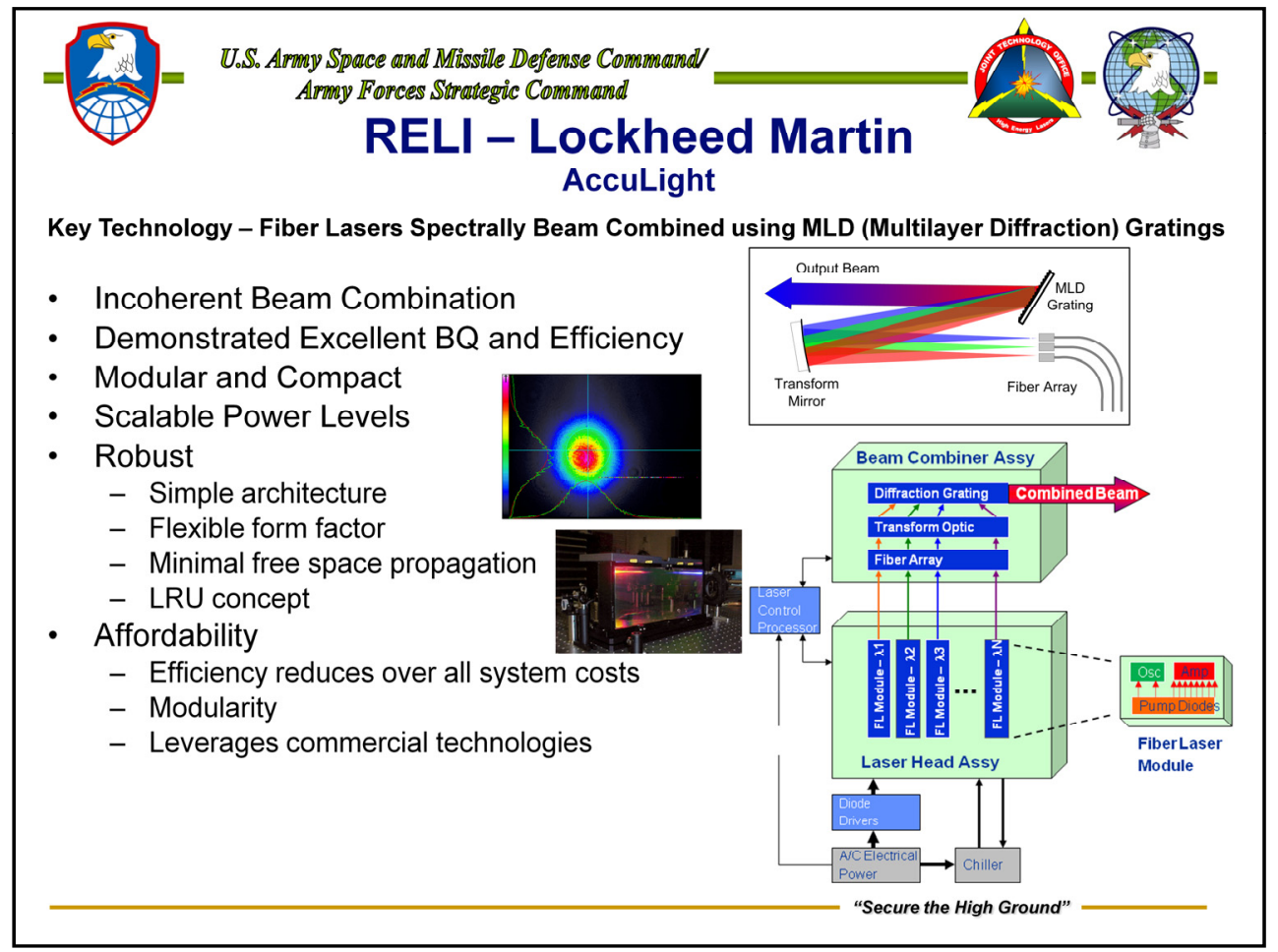

The Lockheed Martin RELI approach is based on their patented and demonstrated Spectral Beam Combining (SBC) of fiber lasers using multilayer dielectric (MLD) gratings. The robustness, high efficiency, simplified packaging, and thermal management of fiber lasers offer significant benefits over other approaches. The MLD, proven to handle $>17 \mathrm{~kW} / \mathrm{cm} 2$ in previous LM tests, allows for all reflective optics permitting scaling to $100+\mathrm{kW}$. The SBC also exhibits graceful degradation compared to coherent combining approaches, without beam quality changes if individual fiber modules degrade or drop out. Also, since the majority of the optical path is in fiber with only a small free-space assembly that must be kept free of contaminants and carefully aligned, the system is well suited to ruggedization for military systems.

LM's architecture has delivered a beam quality of M2 $<1.5$ and $1 \mathrm{~kW}$ output power in a laboratory system using five fiber channels. They also recently demonstrated $32 \%$ electrical efficiency for a fiber laser SBC system. Under an IRAD program LM demonstrated grating performance and survivability at the optical power densities required for a 100-kW weapon.

Beam combining of fiber lasers overcomes the power scaling limits of a single fiber while providing the beam quality (BQ), size, weight, and efficiency advantages of fiber lasers over conventional slab and thin disk solid state lasers. The proposed beam combined fiber laser system provides the core capability for Directed Energy Weapon (DEW) systems for a variety of platforms with the ruggedness, efficiency, size, weight and volume benefits that are unavailable from other electric laser approaches. The Lockheed Martin proposed architecture meets all RELI performance requirements, and the Base and Option programs provide for both early demonstration of these parameters and clear traceability to fieldable military HEL weapon systems. 


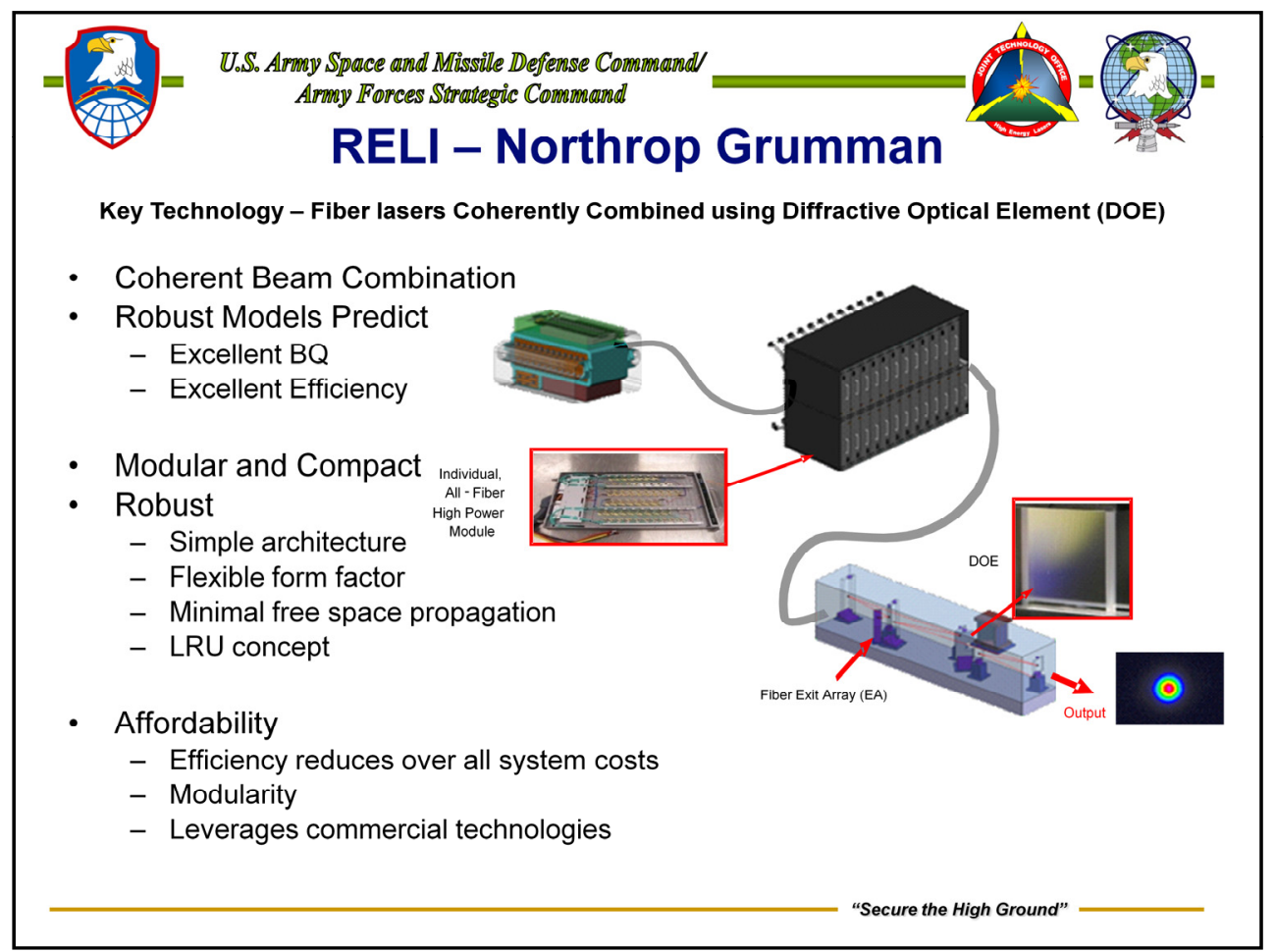

Northrop Grumman's RELI effort is based on innovative and proven technology that advances the state of the art in high power and high efficiency electric lasers. NG's scalable architecture employs a highly efficient, actively phase-locked array of high-power fiber amplifiers. The amplifiers are coherently combined with excellent beam quality using a proven, custom diffractive optical element (DOE). NG projects $23 \mathrm{~kW}$ power in the bucket (PIB) which meets the $22 \mathrm{~kW}$ goal and exceeds the $12 \mathrm{~kW}$ requirement with $95 \%$ margin, in a compact package readily traceable to a field-ready $100 \mathrm{~kW}$ system.

NG's laser concept is inherently rugged and militarily supportable. It is ideally suited to power scaling of fieldable lasers to $100 \mathrm{~kW}$ and beyond and providing military utility for a broad range of platforms and applications. The design provides very high efficiency and excellent beam quality that maximizes the PIB to enable laser weapon systems with enhanced lethality and longer range, for both offensive and defensive missions such as counter-RAM (rockets, artillery, and mortars) or precision strike. The high PIB efficiency in combination with the flexible, highly compact, and light weight fiber-based system enables revolutionary new mission capabilities on limited payload platforms such as unmanned aerial vehicles.

To exceed the RELI objective of improving performance and military utility of stateof-the-art high power electric lasers, NG proposed a highly scalable, efficient, and near-diffraction limited laser system consisting of an actively phase-locked array of high-power Ytterbium (Yb)-doped fiber amplifiers (YDFAs). By means of a custom, highly-efficient DOE, the array of fiber beams are coherently and efficiently combined into a single beam of excellent beam quality $(B Q)$ and spectral brightness. 


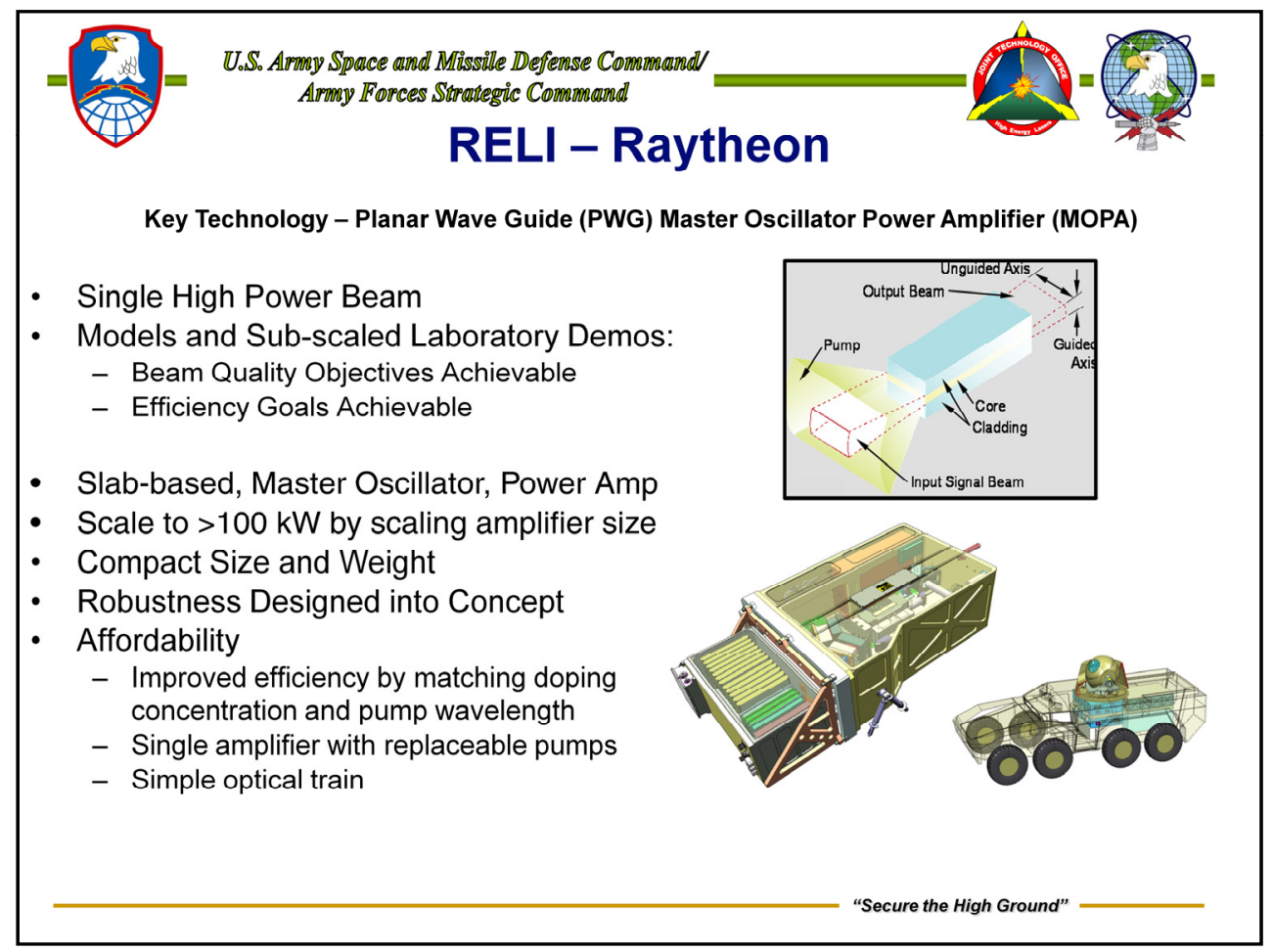

Raytheon's approach is based on a ruggedized 25kW Yb:YAG Planar WaveGuide (PWG) Master Oscillator Power Amplifier (MOPA) with a 200W fiber laser $\mathrm{MO}$ and a single PWG amplifier boosting the output power to $>27 \mathrm{~kW}$ with $>30 \%$ efficiency. The $25 \mathrm{~kW}$-class laser exceeds the RELI Power In the Bucket (PIB) threshold goal of $12 \mathrm{~kW}$ in a $1.5 \mathrm{~N} / \mathrm{D}$ far-field bucket and approaches the RELI PIB stretch goal of $22 \mathrm{~kW}$ with a RELI-defined PIB Efficiency (PIBE) of 18-22\%. This simple architecture can be scaled to $>100 \mathrm{~kW}$ without adding amplifiers by scaling the size of the PWG. The single beamline approach avoids complex beam combining or phasing of multiple beams. The Size, Weight, and Power (SWAP) of the ruggedized package are compatible with integration into a single tactical vehicle.

Because of the importance RELI has placed on efficiency, beam quality, power, and ruggedized packaging, Raytheon has started with the end goal in mind to develop the intermediate demonstration concepts and the risk reduction analyses and experiments that need to be conducted in order to get there. Raytheon's vision is a $100 \mathrm{~kW}$ ruggedized PWG laser that is simple (i.e., has a single amplifier) with excellent beam quality (i.e., < 2.0) that has very high efficiency (i.e., > 30\%) and has adaptive optics built in to provide beam cleanup and atmospheric compensation so as to achieve long engagement ranges so large areas can be defended with fewer systems. 


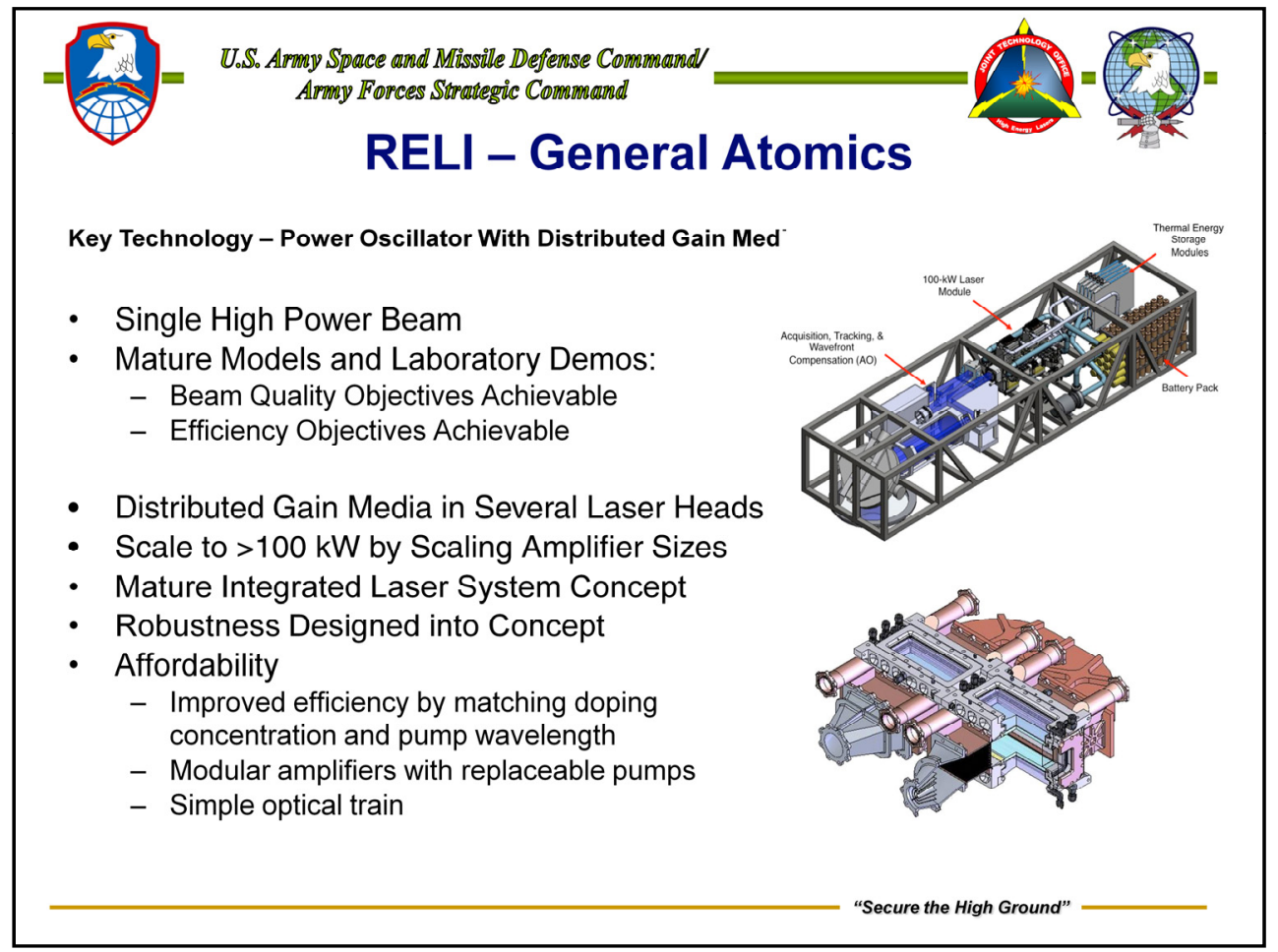

GA's approach for RELI is based on their demonstrated Distributed Gain technology. Distributed Gain is unique among other approaches in that it provides a remarkably compact and lightweight approach to weapon class lasers enabling real implementation on tactical platforms. The RELI system concept incorporates two unit cells. To date, they have constructed a single unit cell. Laboratory experiments to date have shown exceptional laser performance with higher than expected laser power output and excellent beam quality. While the RELI desired goal for size and weight has already been demonstrated, the electrical to optical efficiency is the primary emphasis of the GA RELI program.

GA's approach to meet the ruggedness, efficiency and laser performance objectives of RELI is to create a new, High Efficiency version of their distributed gain media laser. In particular, they will keep the features of the current system which enable small size and weight with good beam quality but will change the laser medium, pump diodes and head design to achieve the RELI efficiency goal of $\sim 30 \%$ with an $1.5 \mathrm{l} / \mathrm{D}$ half-angle encircled power output of $100 \mathrm{~kW}$. While the basic architecture on which our RELI design is based has been demonstrated in the laboratory, the overall technology readiness level with respect to RELI remains well below TRL 6 at this time. 


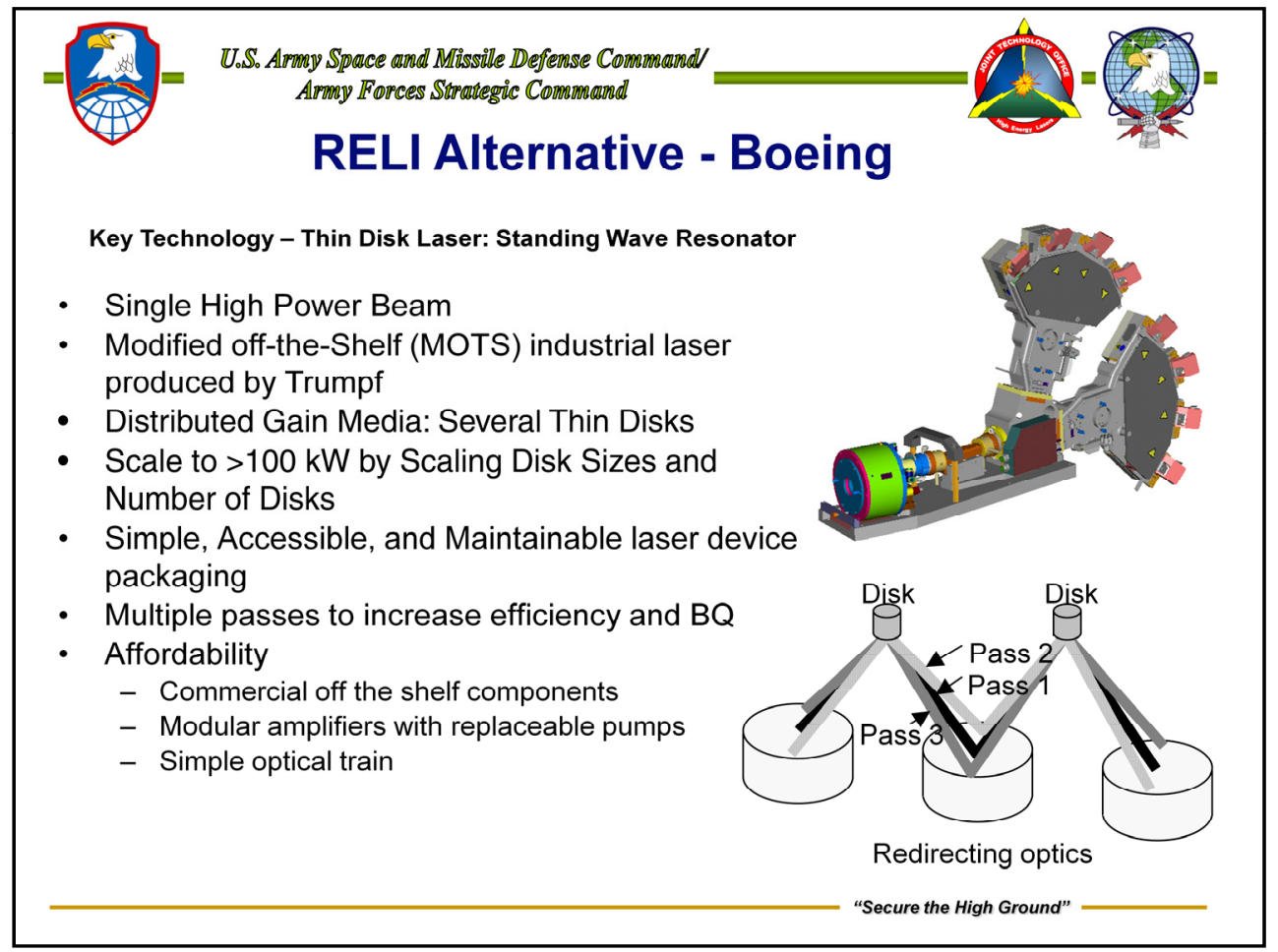

Boeings approach is a Thin Disk Laser. TDL's are interesting because they offer an excellent alternative to deliver a robust, affordable solid state laser, and there has been significant industry investment based on industrial-grade TDL's. Laboratory demonstrations have recently produced power levels of greater than $25 \mathrm{~kW}$, with good beam quality, and good E-O efficiency.

With further design optimization, higher E-O efficiency should be attainable, which further improves specific brightness and overall laser weapon system performance. With recent test results that show the thin disk area can be significantly scaled without significant Amplified Stimulated Emission (ASE), this provides a low-risk technology development path to $100 \mathrm{~kW}$ or greater. The expected potential improvements will support the RELI Power In the Bucket (PIB) objective without the need for high-risk technology scaling. Developing and demonstrating these improvements constitutes the core of the Thin Disk Laser (TDL) High Brightness Laser Development program. 


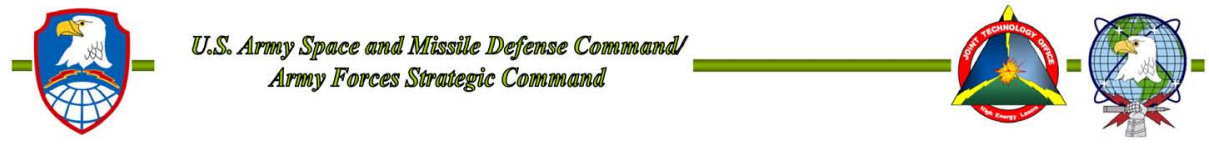

\section{Beam Control Technology}




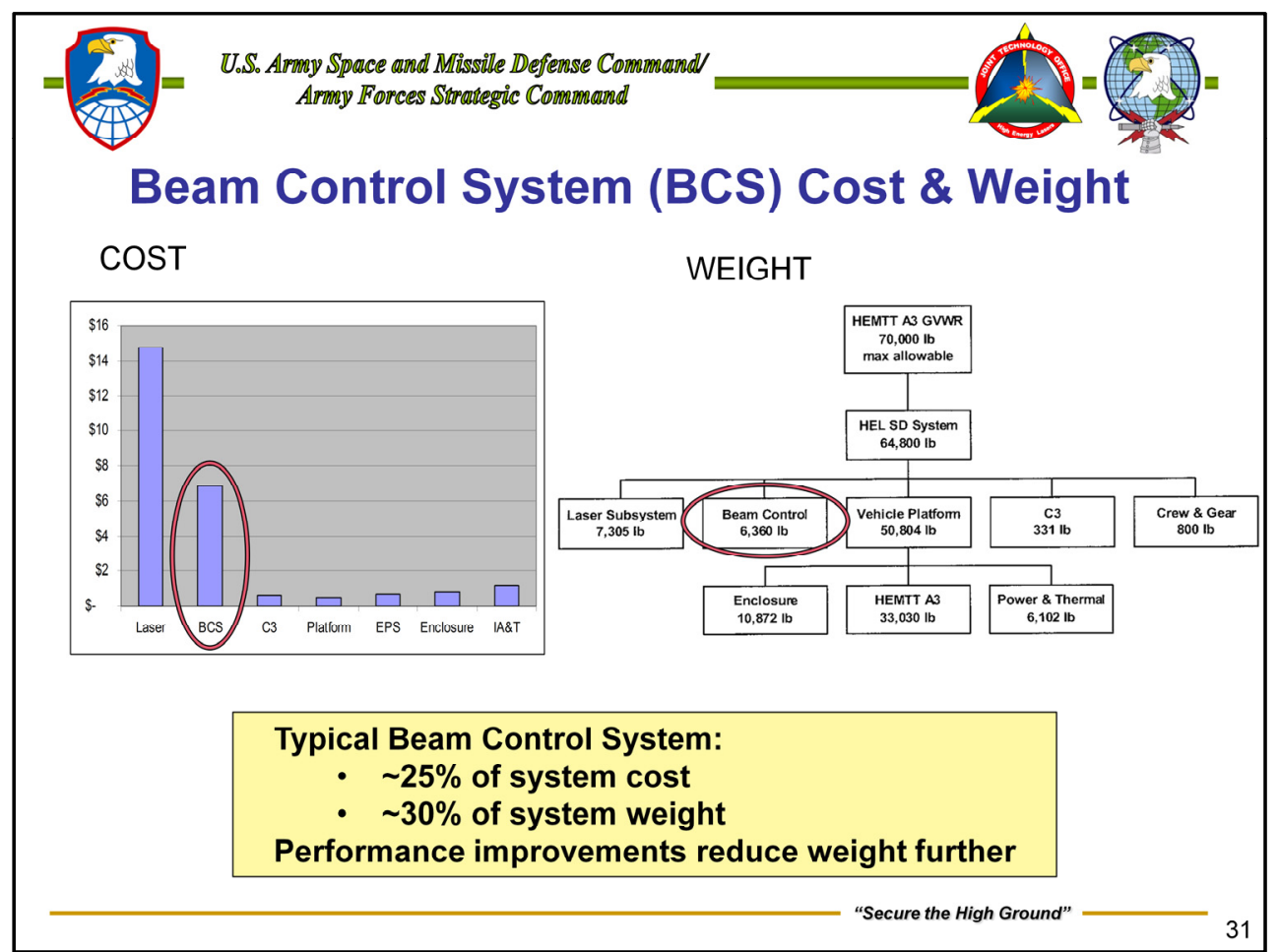

From substantial analyses conducted under the HELTD program, the cost and weight of the beam control system are major factors in the development of a HEL weapon system. This chart indicates that approximately $25 \%$ of the system development cost may be devoted to the BCS, and $\sim 30 \%$ of the system weight can be attributed to the BCS. With substantial improvement in BCS technologies and approaches, the reductions in cost and weight realized will greatly improve the compatibility of modern BCS's with tactical platforms. That is the purpose of the Light-Weight Beam Director (LWBD) program. 


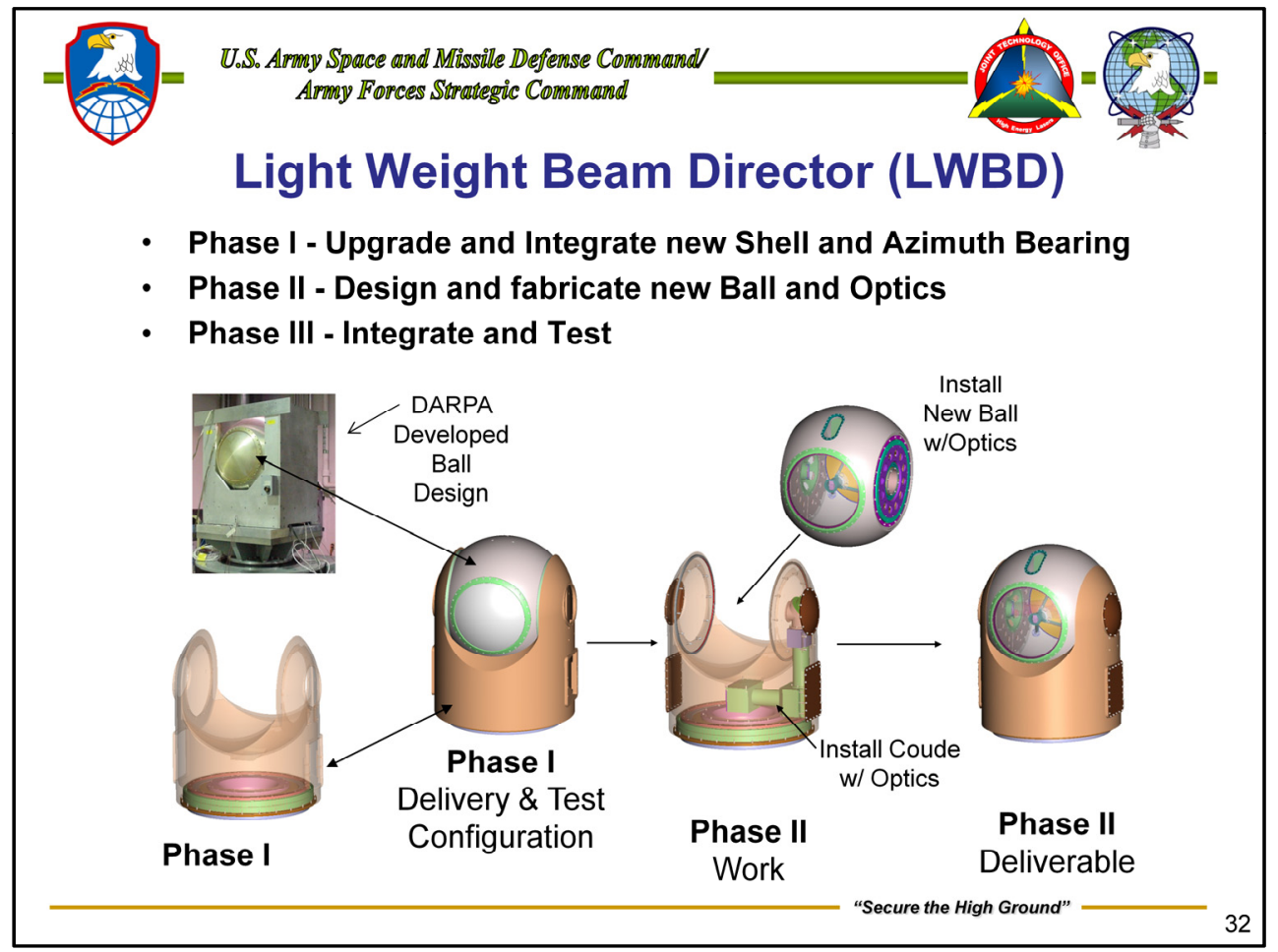

The Light-Weight Beam Director (LWBD) development effort is being conducted in phases. The initial phase was actually conducted under a DARPA program, resulting in the development of a "ball telescope" structure that was used to conduct early performance testing in the laboratory of gimbal motion. When DARPA elected not to further develop the concept, the HEL JTO and Army elected to take the project and continue the development in phases.

The Phase I activity is nearing completion, with the delivery and testing of a "shell" designed to add an azimuth range of motion and accommodate the ball telescope design as a line replaceable unit (LRU).

As funding permits, the Phase II work planned will design and fabricate a new ball with refractive optics and install a Coude path in the existing shell. The phase II deliverable will be the integrated system ready for further testing in conjunction with a high average power solid state laser. 


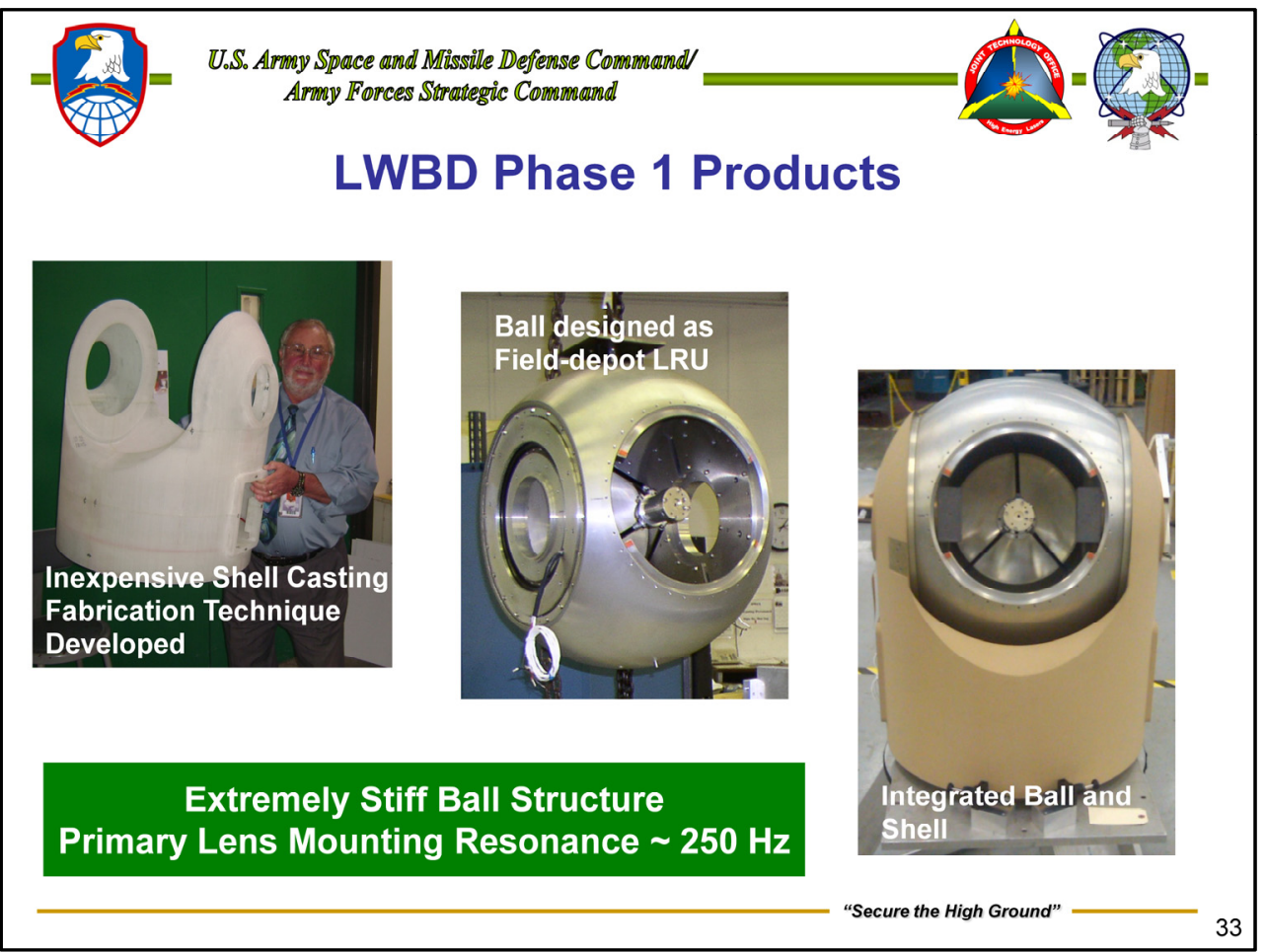

Shown here are the products of the Phase 1 effort. 


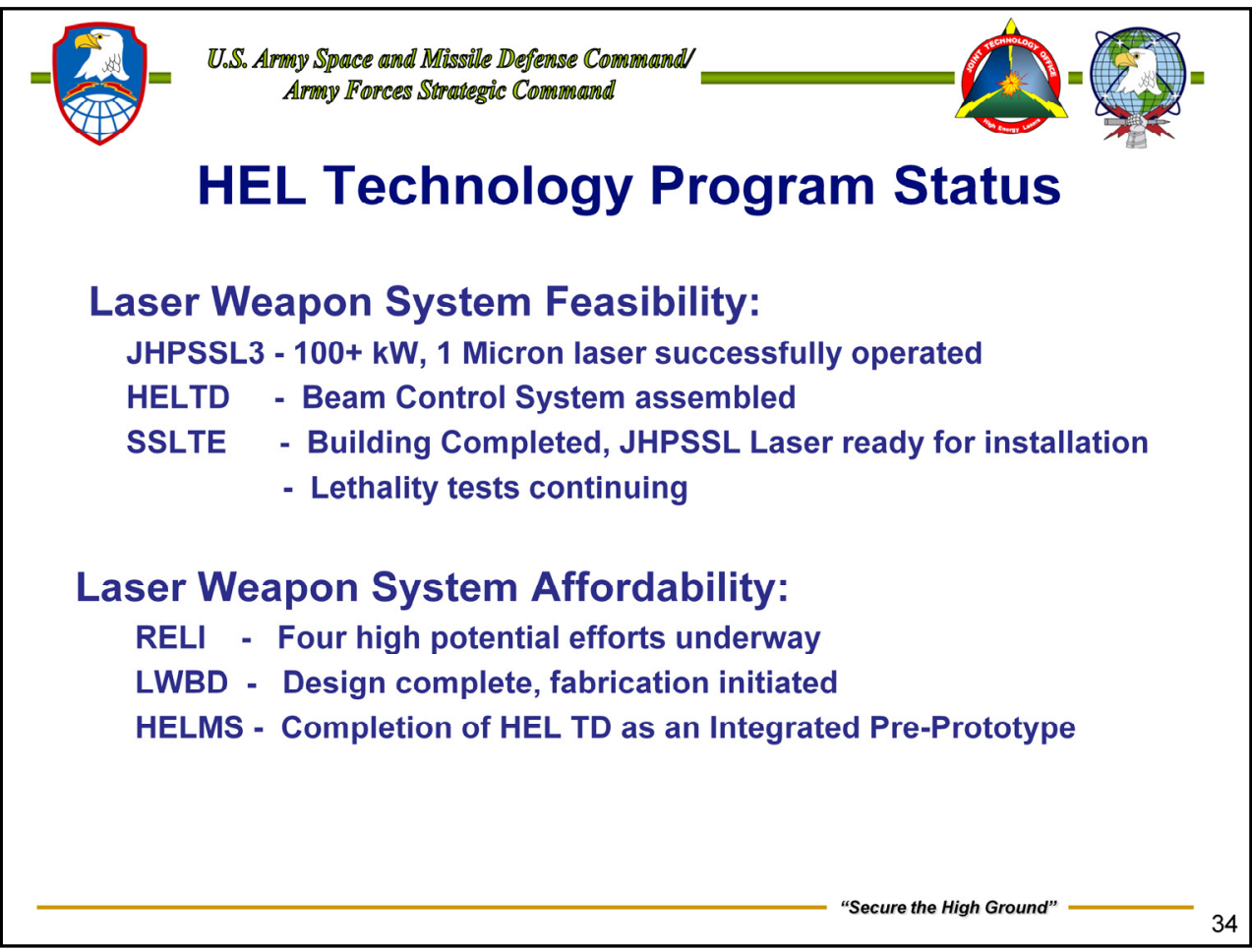

I would like to summarize where we are in the development of High-Energy Laser weapons systems. The feasibility of shooting down rockets, artillery, and mortars (RAM) targets with a high energy chemical laser was demonstrated with the Tactical High Energy Laser (THEL) program over 10 years ago. Although the THEL demonstration was success, the logistics and safety issues associated with the chemicals used to power the laser made it impractical for use as a fielded weapons system for the Army. To overcome these issues, emphasis shifted towards the development of solid-state lasers as they are electrically powered.

At that time, a joint effort to advance the state-of-the-art of SSL technology was initiated by the OSD HEL-JTO and the services. The results of this effort have largely shown that obtaining weapon performance from a solid state laser (SSL) is feasible. The Joint High Power Solid State Laser (JHPSSL) program successfully demonstrated two laboratory SSLs with $>100 \mathrm{~kW}$ output, while simultaneously meeting other performance criteria in the laboratory.

Other activities to demonstrate other aspects of a SSL based weapon system have also been initiated. The THEL beam director has been modified to accept SSL wavelengths to enable near term propagation and lethality testing. The installation of adaptive optics under the Beam Control Integrated Experiment (BCID) to improve range is underway. Work is in process on the command, control, and communications system (C3) and the beam transport between the laser and THEL beam director. In addition, a "ruggedized" beam control system that will be mounted on a tactical military vehicle is being developed by the High Energy Laser Technology Demonstrator (HEL TD) program, with low power field testing scheduled for late 2011 and high power testing in 2012 using a JHPSSL device.

The effort to improve the affordability of HEL weapon systems is also now underway. The Robust Electric Laser Initiative (RELI) will improve the technology required to significantly improve efficiency, thereby substantially reducing the volume and weight parameters for SSL weapons to be demonstrated with the HELMS. Consequently, by reducing prime power and thermal management subsystem requirements, weapon system affordability will also be significantly improved. 


\section{High Power Microwave (HPM) Technologies}




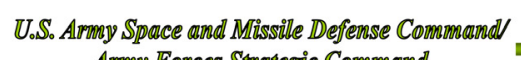

Alpmy IForces Strategic Command

\section{HPM Weapon Systems}

\section{Applications}

- Disrupt/Defeat $C^{3} \mathbf{I}$

- Disrupt / Defeat EO/IR Sensors Used to Detect, Track and Engage Systems

- Standoff Mine Neutralization and Explosive Ordnance Disposal (e.g. Improvised Explosive Devices (IEDs))

- Ultra-Precision Strike - Kill / Disable Targets with No (Minimal) Collateral Damage

\section{Systems}

- Grenades

- Rockets/Missiles

- Airborne - UAV or Helicopter

- Ground Vehicles

SMDC is developing both explosive and non-explosive pulsed power for HPM systems. Our interest in explosive pulsed power is primarily driven by the size of the delivery platform that we have to work with. 


\section{U.S. Alpmy Space andl Misssile Defense CommerandV Alrmy Forces Starategic Command \\ Pulse Power Requirements for HPM Systems}

- SMDC is developing both explosive and non-explosive pulse power for HPM and Laser systems

- Factors that determine which type of pulsed power is suitable for a given platform or application are:

- Desired Effects/Lethality

- Required Repetition Rate

- Platform Volume and Weight Constraints

- SMDC has focused much of its pulsed power development on explosive pulsed power.

- Reliable explosive pulsed power generators that provide consistent outputs have been developed for different applications.

"Secure the High Ground"

Factors that determine which type of pulsed power to use are

1. Volume available in the platforms of interest. Most army missile warheads have very limited volume, which means we are forced to use explosive pulsed power.

2. Desired lethality - a warhead with enhanced lethality (HPM plus blast and fragmentation) or less-than-lethal (HPM only).

3. Repetition rate - a single or short train of pulses vs. a long train of pulses. Rep rate is preferable, but not always possible depending on the platform and application.

4. Desired effects - burn out vs. upset 


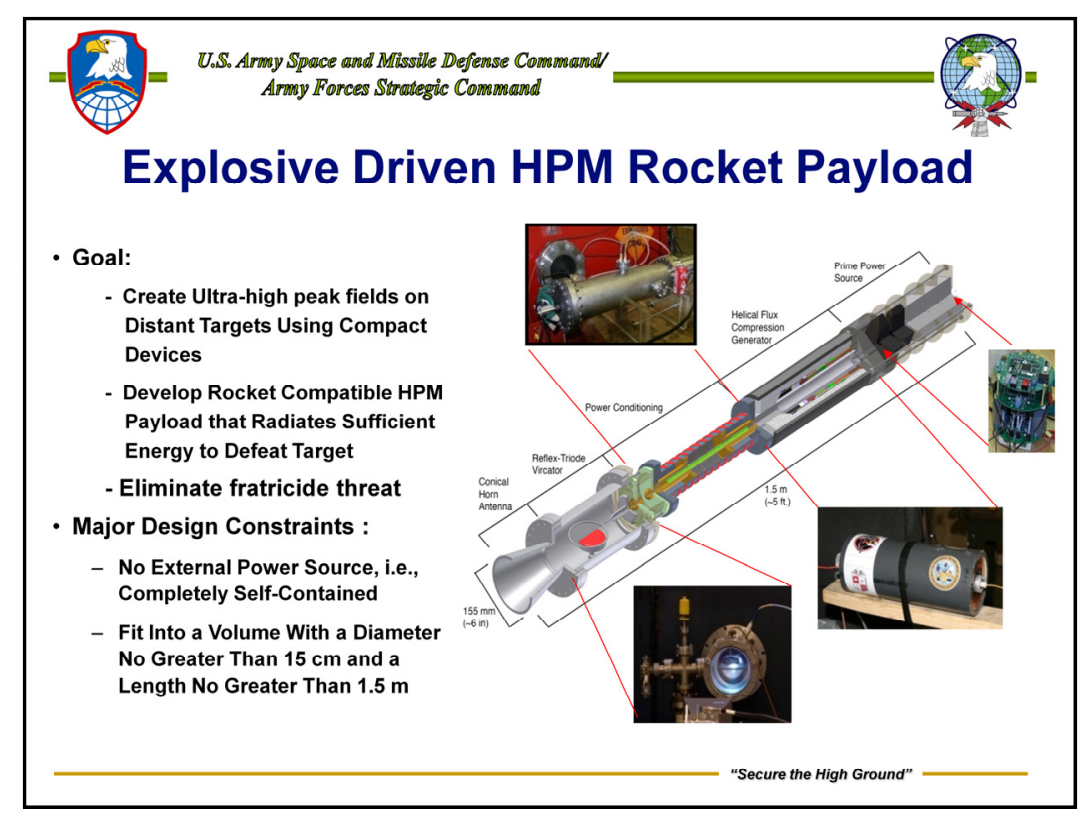

Here is an example of where we use explosive pulsed power to drive a HPM tube (Virtual Cathode Oscillator - Vircator). In April, 2009, Texas Tech University successfully demonstrated an autonomous explosive driven HPM system at Redstone Arsenal. By autonomous, we mean the system is completely self-contained; i.e., the system has no external cables. The system consists of four major components.

The prime power source, which provides the seed magnetic field to the Flux Compression Generator (FCG) and the firing pulse to the detonators in the FCG.

The Flux Compression Generator, which amplifies the energy from the seed source and delivers it to the power conditioning stage.

The power conditioning stage which shortens the rise time and increases the peak voltage of the pulse produced by the FCG. FCGs are inherently low voltage power supplies and the power conditioning stage is needed to increase the voltage to that required to drive the microwave tube.

The fourth stage is the microwave tube, which is a virtual cathode oscillator. Work is now ongoing to make this a sealed vacuum tube with a shelf life of 10 years. It should be noted that this system was designed, built, and tested by graduate students at Texas Tech and the University of Missouri.

The main objective of this effort is train students to work with explosive pulsed power, high power microwaves and high voltage engineering. 


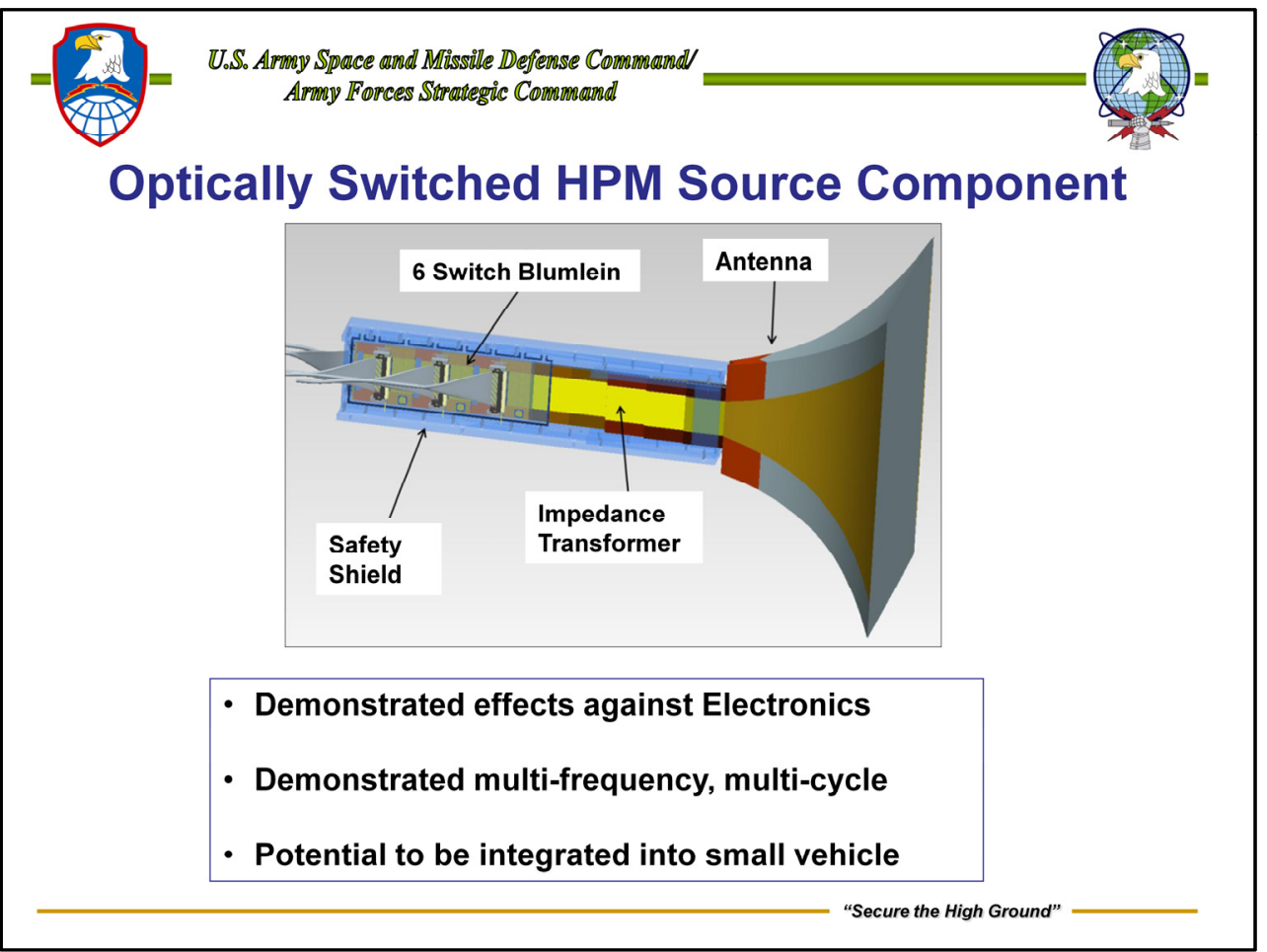

- Digital Synthesis of RF using Optical Switches to discharge Blumlein line

- 1 charge line. 2 use laser pulse to close switches. (fast \& time precise) allows for control of pulse shape.

- System is array of individual lines. Phase array concept, potential of airbreakdown electric fields

- Modular and steerable 
U.S. Alromy Space and Misssille Defense CommondV Armon Forces Starategic Command

\section{Integrated HPM Source Array Payload}

Single Optically Switched RF Source

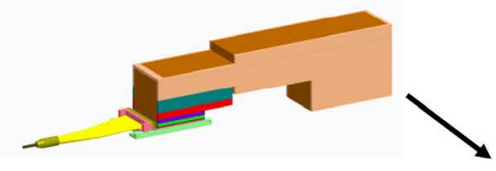

$\sim 12 \times 2 \times 4$ inches

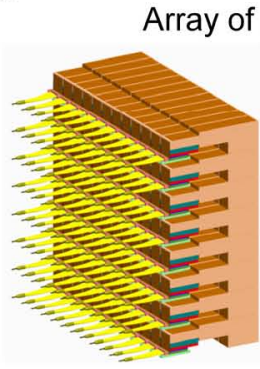

- Array is scalable in $x, y$, and $z$ depending upon energy requirements

- Electronically steerable

- Frequency agile

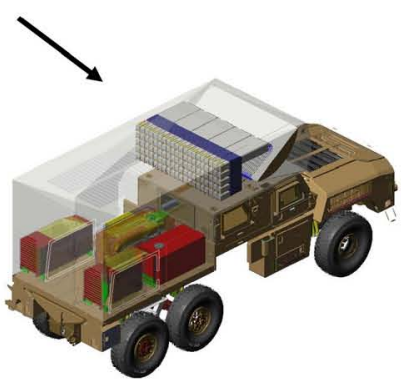




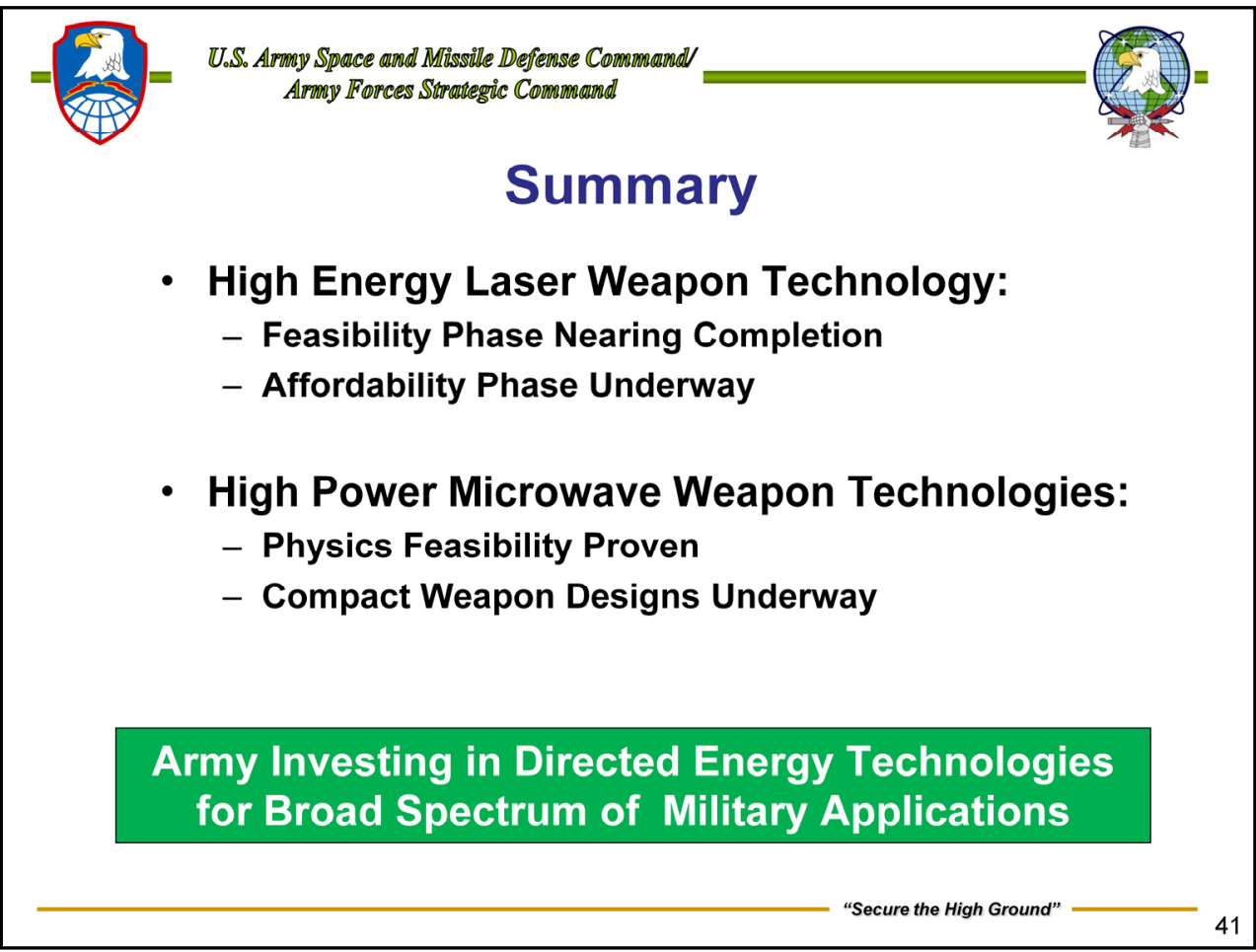

I would like to summarize where we are in the development of High-Energy Laser weapons systems. The feasibility of shooting down rockets, artillery, and mortars (RAM) targets with a high energy chemical laser was demonstrated with the Tactical High Energy Laser (THEL) program over 10 years ago. Although the THEL demonstration was success, the logistics and safety issues associated with the chemicals used to power the laser made it impractical for use as a fielded weapons system for the Army. To overcome these issues, emphasis shifted towards the development of solid-state lasers as they are electrically powered.

At that time, a joint effort to advance the state-of-the-art of SSL technology was initiated by the OSD HEL-JTO and the services. The results of this effort have largely shown that obtaining weapon performance from a solid state laser (SSL) is feasible. The Joint High Power Solid State Laser (JHPSSL) program successfully demonstrated two laboratory SSLs with $>100 \mathrm{~kW}$ output, while simultaneously meeting other performance criteria in the laboratory.

Other activities to demonstrate other aspects of a SSL based weapon system have also been initiated. The THEL beam director has been modified to accept SSL wavelengths to enable near term propagation and lethality testing. The installation of adaptive optics under the Beam Control Integrated Experiment (BCID) to improve range is underway. Work is in process on the command, control, and communications system (C3) and the beam transport between the laser and THEL beam director. In addition, a "ruggedized" beam control system that will be mounted on a tactical military vehicle is being developed by the High Energy Laser Technology Demonstrator (HEL TD) program, with low power field testing scheduled for late 2011 and high power testing in 2012 using a JHPSSL device.

The effort to improve the affordability of HEL weapon systems is also now underway. The Robust Electric Laser Initiative (RELI) will improve the technology required to significantly improve efficiency, thereby substantially reducing the volume and weight parameters for SSL weapons to be demonstrated with the HELMS. Consequently, by reducing prime power and thermal management subsystem requirements, weapon system affordability will also be significantly improved. 
2.

\section{Backup}




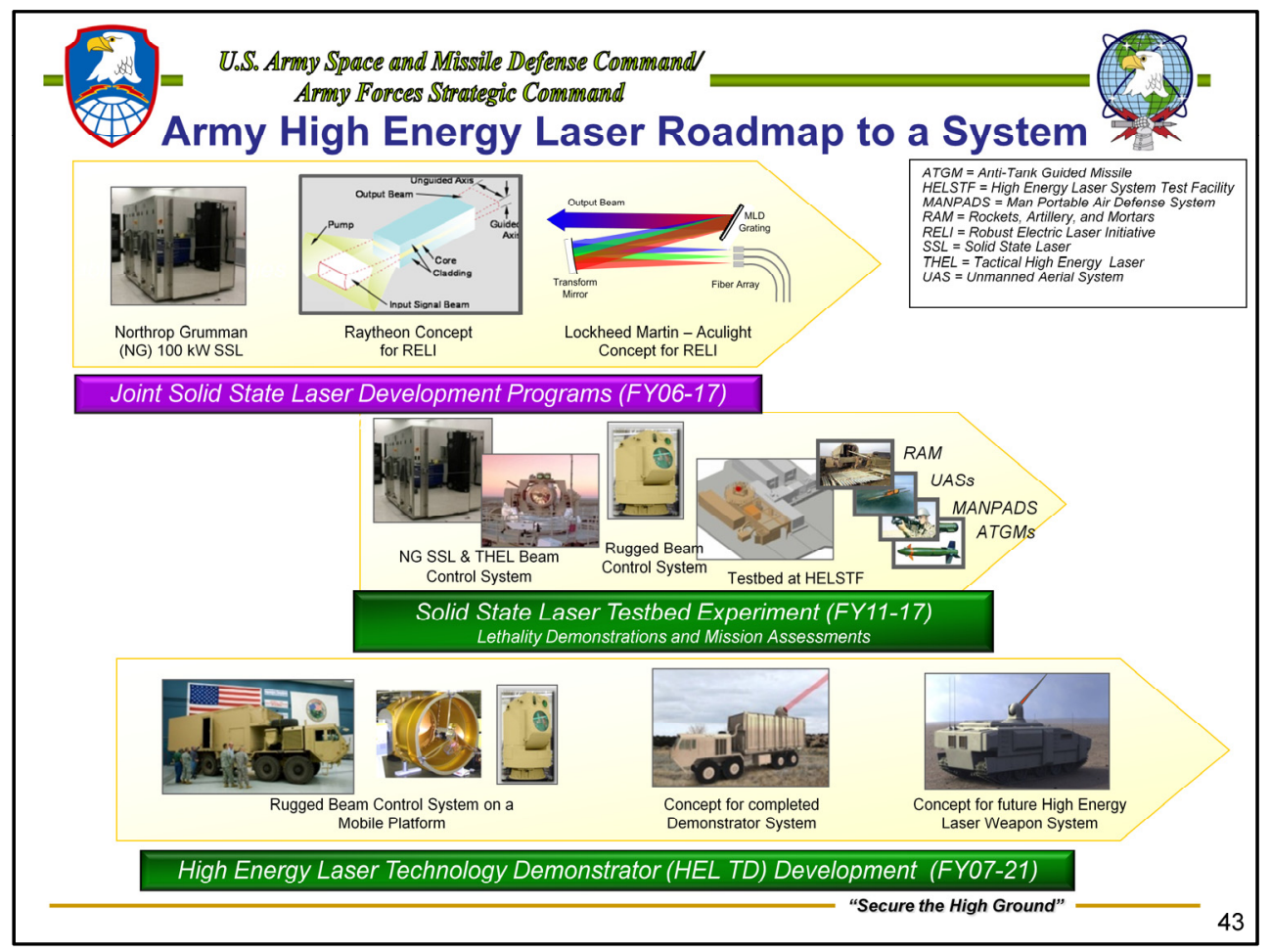

The Army's HEL Science and Technology (S\&T) Program is summarized here. Through joint solid state laser development programs, the DoD has advanced the power of SSLs from $1 \mathrm{~kW}$ to $>100 \mathrm{kWs}$ over the past 10 years. The goals of the initial phases of the Joint High Power Solid State Laser (JHPSSL) program were to develop 25kW class laboratory devices with near diffraction limited beam quality and long runtime. Under the JHPSSL Phase 3 program, SMDC/ARSTRAT led the development of two alternative solid state laser laboratory devices in conjunction with the OSD High Energy Laser Joint Technology Office (HEL JTO), Army Research Laboratory, Air Force Research Laboratory, and the Office of Naval Research. The JHPSSL Phase 3 program successfully demonstrated in FY09 and FY10 alternative 100kWclass, diode-pumped solid-state lasers with architectures that are favorable for tactical weapon applications. Follow-on joint SSL development activities, such as the Robust Electric Laser Initiative (RELI), are focusing on improved efficiency to enable compatibility with future Army tactical vehicles and reduced overall weapon system cost.

The Army S\&T program includes the development of the Solid State Laser Testbed Experiment (SSLTE) at the High Energy Laser Systems Test Facility (HELSTF) to conduct lethality testing, shootdown demonstrations, and mission assessments. The SSLTE will become operational in late FY11.

The Army S\&T program also includes the High Energy Laser Technology Demonstrator (HEL TD) program to demonstrate in a relevant operational environment its capability to counter RAM and other targets. The HEL TD will consist of an SSL, beam control, electrical power supply, thermal management, fire control and communications elements on a wheeled tactical platform.

The Army S\&T Program is also conducting basic and applied research on enabling technologies in the areas of advanced beam control and power/thermal management components. Component technologies are being developed by TARDEC and ARL, including programmable pulse power supplies, solid state switches and capacitors, power electronics coolant loops, and intelligent power management hardware and software. These activities, along with SSLTE and HEL TD testing and evaluation, will support an informed decision for transitioning to acquisition of Army laser weapon systems to provide enhancements to the Army future force.

Key Milestones: Utilize Northrop Grumman JHPSSL $100 \mathrm{~kW}$ laser at HELSTF and integrate with the THEL Beam Director (modified for operation at $1.06 \mathrm{um}$ ) for propagation and lethality testing and mission area assessments beginning in 2012; Demonstrate 25kW next generation laboratory device(s) in 2015 (RELI); Complete mobile HEL TD Beam Control System (CDR completed July 2009) and conduct low power tests in Summer 2011 against RAM targets at HELSTF; Inject the JHPSSL $100 \mathrm{~kW}$ laser (laser fixed site) into the mobile HEL TD beam control system and perform high power C-RAM tests in 2012; Begin development of a high power ruggedized laser in 2013 for integration into the mobile HEL TD 


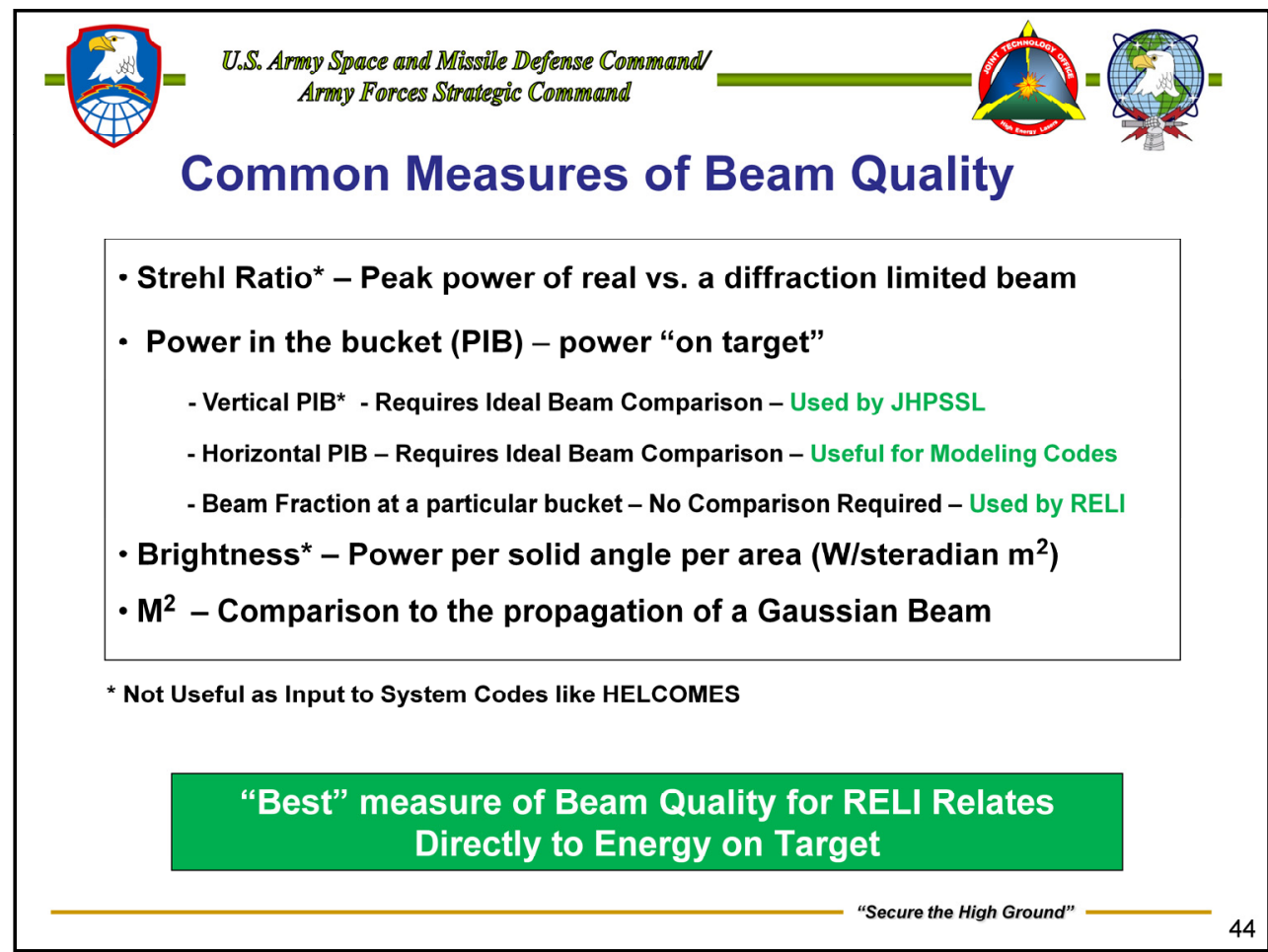

In order to conduct near term (FY11) mission area assessments of solid state laser capabilities, SMDC recommended and received ASAALT approval to develop the Solid State Laser Testbed Experiment (SSLTE) at the High Energy Laser Systems Test Facility (HELSTF) utilizing 6.2 funds. The solid state laser testbed utilizes existing assets, including the Northrop Grumman JHPSSL $3100 \mathrm{~kW}$ device and the THEL system Beam Control System, modified for SSL wavelengths.

The SSLTE will provide risk reduction activities for the High Energy Laser Technology Demonstrator, and establish a weapon power testbed available for use by the Army, other Services, and DoD to conduct developmental testing, propagation experiments, and lethality experiments. 

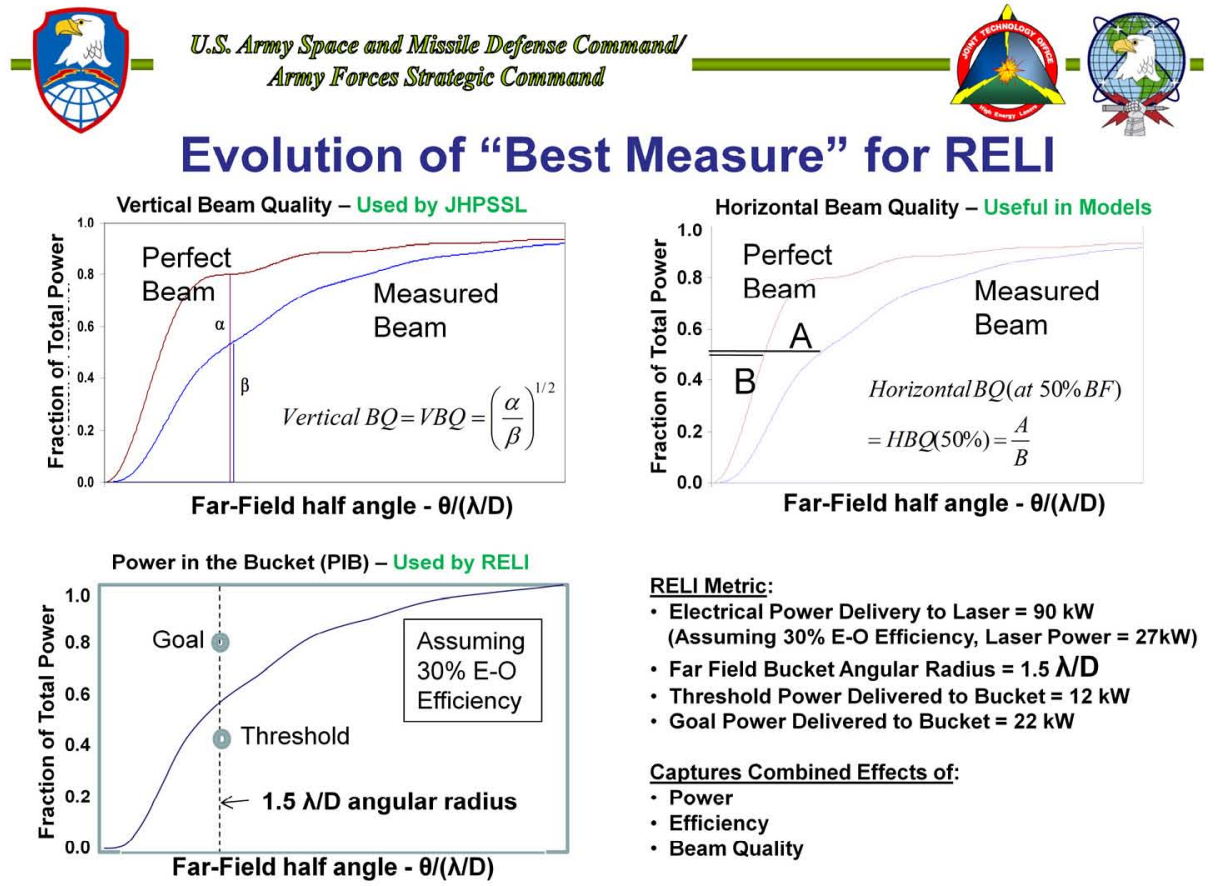

RELI Metric:

- Electrical Power Delivery to Laser $=90 \mathrm{~kW}$

(Assuming 30\% E-O Efficiency, Laser Power $=27 \mathrm{~kW}$ )

- Far Field Bucket Angular Radius = 1.5 N/D

- Threshold Power Delivered to Bucket $=12 \mathrm{~kW}$

- Goal Power Delivered to Bucket $=22 \mathrm{~kW}$

Captures Combined Effects of:

- Power

- Efficiency

- Beam Quality 